Article

\title{
Granulation of Hypernetwork Models under the q-Rung Picture Fuzzy Environment
}

\author{
Anam Luqman ${ }^{1}$, Muhammad Akram ${ }^{1, *(1)}$ and Ali N. A. Koam ${ }^{2}$ (]) \\ 1 Department of Mathematics, University of the Punjab, New Campus, Lahore 54590, Pakistan; \\ anamluqman7@yahoo.com \\ 2 Department of Mathematics, College of Science, Jazan University, New Campus, P.O. Box 2097, Jazan 45142, \\ Saudi Arabia; akoum@jazanu.edu.sa \\ * Correspondence: m.akram@pucit.edu.pk
}

Received: 3 May 2019; Accepted: 27 May 2019; Published: 1 June 2019

\begin{abstract}
In this paper, we define $q$-rung picture fuzzy hypergraphs and illustrate the formation of granular structures using $q$-rung picture fuzzy hypergraphs and level hypergraphs. Further, we define the $q$-rung picture fuzzy equivalence relation and $q$-rung picture fuzzy hierarchical quotient space structures. In particular, a $q$-rung picture fuzzy hypergraph and hypergraph combine a set of granules, and a hierarchical structure is formed corresponding to the series of hypergraphs. The mappings between the $q$-rung picture fuzzy hypergraphs depict the relationships among granules occurring at different levels. The consequences reveal that the representation of the partition of the universal set is more efficient through $q$-rung picture fuzzy hypergraphs and the $q$-rung picture fuzzy equivalence relation. We also present an arithmetic example and comparison analysis to signify the superiority and validity of our proposed model.
\end{abstract}

Keywords: $q$-rung picture fuzzy set; $q$-rung picture fuzzy hypergraphs; granular computing; algorithms

\section{Introduction}

Granular computing $(\mathrm{GrC})$ is defined as an identification of techniques, methodologies, tools, and theories that yield the advantages of clusters, groups, or classes, i.e., the granules. The terminology was first introduced by Lin [1]. The fundamental concepts of $\mathrm{GrC}$ are utilized in various fields of research, including rough set theory, machine learning, fuzzy set theory, and artificial intelligence. Different models have been proposed to study the numerous issues occurring in $\mathrm{GrC}$, including classification of the universe, illustration of granules, and the identification of relations among granules. For example, the procedure of problem solving through $\mathrm{GrC}$ can be considered as distinct descriptions of the problem at multiple levels, and these levels are linked together to construct a hierarchical space structure. Thus, this is a way of dealing with the formation of granules and the switching between different granularities. Here, the term "hierarchy" implies the methodology of hierarchical analysis in solving complicated problems and human diversions [2]. To understand this methodology, let us consider an example of national administration in which the complete nation is subdivided into various provinces. Further, divide every province into various divisions similarly. The human activities and problem solving involve the simplification of the original complicated problem by ignoring some details rather than thinking about all points of the problem. This rational model is then further sifted till the issue is completely resolved. Thus, we resolve and interpret the complex problems from the weaker grain to the stronger one, or from the highest rank to lowest, or from universal to particular. This technique is called hierarchical problem solving. It is further acknowledged that the hierarchical strategy is the only technique that is used by humans to deal with complicated problems, and it enhances the competency and efficiency. This strategy is also known as multi-GrC. Yang et al. [3] 
proposed a partition-based knowledge distance based on the Earth Mover's Distance (EMD) and proved that their knowledge distance is equivalent to the binary granule-based knowledge distance (BGKD) but is more intuitive than the BGKD.

Granulation for simple graphs was firstly introduced by Stell [4]. He demonstrated the different kinds of granulation for simple graphs and discussed the connection of vague graphs with granulation. Chen and Zhong [5] extracted the granular structures using simple graphs. They developed the granular structure models based on three types of elements in a simple graph, i.e., vertices, edges, and their combination. In 2016, Chiaselotti et al. [6] studied some interesting concepts of simple graphs, which correspond to GrC. Further, they applied the theoretical concepts of their work to three families of graphs. Micro and macro models of granular computing based on the indiscernibility relation were studied by Bisi et al. [7]. They investigated the indiscernibility structures of the knowledge representation system and developed three hypergraphic structures, including the discernibility hypergraph, the reduct, and the essential hypergraph.

Hypergraphs, as an extension of classical graphs, experience various characteristics, which are the foundation of many methodologies and appear very effective and useful in many fields, including problem solving, declustering, and databases [8]. The formation of hypergraphs is the same as that of granule structures, and the relations between the vertices and hyperedges of hypergraphs can depict the relationships of granules and objects. A hyperedge can contain $n$ vertices and therefore represents $n$-ary relations, and hence, a hypergraph can provide a more effective analysis and description of granules. Many researchers have used hypergraph methods to study the clustering of complex documentation by means of $\mathrm{GrC}$ and investigated the database techniques [9,10]. Chen et al. [11] proposed a model of $\mathrm{GrC}$ based on a crisp hypergraph. They related a crisp hypergraph to a set of granules and represented the hierarchical structures. They proved their hypergraphic model as a visual description of GrC.

Zadeh's [12] fuzzy set (FS) has acquired greater attention by researchers in a wide range of scientific areas, including management sciences, robotics, decision theory, and many other disciplines. Atanassov [13] originated the notion of the intuitionistic fuzzy set (IFS), which is distinguished by a positive degree $(T)$ and negative degree $(F)$ satisfying the requirement that the summation of both membership degrees should not exceed one and is considered as an extension of FSs. However, in many practical decision-making problems, the sum of positive and negative degrees may not be less than one, but the sum of their squares may be less than one. To handle such a type of difficulty, Yager [14] gave the concept of Pythagorean fuzzy sets (PYFSs) as a generalization of IFSs. The two membership degrees in PYFSs are needed to fulfill the requirement $T^{2}(x)+F^{2}(x) \leq 1$, which shows that PYFSs have expanded space to assign the orthopair as compared to IFSs. PYFS can deal with various real-life problems more effectively, but still, there are cases that cannot be handled using this set. Take an example: the degrees of truth and falsity suggested by a decision maker are 0.8 and 0.9 , respectively. Then, the problem can never be handled by means of PYFS as $0.8^{2}+0.9^{2}=1.45>1$. In order to deal with such types of cases, Yager [15] introduced the idea of the $q$-rung orthopair fuzzy sets ( $q$-ROFSs). A $q$-ROFS is represented by means of two membership degrees: one is truth and the other is falsity, with the characteristic that the summation of the $q$ th power of the positive degree and the $q$ th power of the negative degree should not be greater than one. In 2014, Cuong [16] introduced picture fuzzy sets (PFSs), which generalize the concepts of FSs and IFSs. He also studied some operations on PFSs. Li et al. [17] proposed the extension of $q$-ROFSs and PFSs as $q$-rung picture fuzzy sets ( $q$-RPFSs). A $q$-RPFS expresses the impartial behavior in the form of the neutral degree and also relaxes the restriction of PFSs. The limitation of $q$-RPFS implies that $T(x)^{q}+N(x)^{q}+F(x)^{q} \leq 1,(q \geq 1)$, and this is considered as the most prominent characteristic of $q$-RPFS. The space of depiction of acceptable triplets can be widened by taking the larger values of $q$ into consideration; for example, if an agent provides the positive, negative, and neutral degrees as $0.8,0.6$, and 0.7 , respectively. It is clearly seen that $0.8+0.6+0.7 \geq 1$ and $0.8^{2}+0.6^{2}+0.7^{2} \geq 1$. Thus, it is appropriate to apply $q$-RPFS since $0.7^{q}+0.5^{q}+0.6^{q} \leq 1$ for sufficiently large $q$. Moreover, the interdependency of functions $T, N$, and $F$ makes this concept closer to real-world applications. Specific types of Pythagorean fuzzy graphs and 
applications to decision-making were discussed by Akram et al. [18]. Recently, a novel description on edge-regular $q$-rung picture fuzzy graphs with applications was proposed by Akram et al. [19].

To combine the benefits of both fuzzy sets and hypergraphs, Kaufmann [20], in 1977, defined fuzzy hypergraphs. Fuzzy hypergraphs were redefined and generalized by Lee-Kwang and Keon-Myung [21]. A valuable contribution to fuzzy graphs and fuzzy hypergraphs was given in [22]. Many researchers have explored the formation of granules/granular structures using hypergraphs in various fields, but there are many graph theoretic problems that may contain uncertainty and vagueness. To overcome the problems of uncertainty in models of $\mathrm{GrC}$, Wang and Gong [23] studied the construction of granular structures by means of fuzzy hypergraphs. They concluded that the representation of granules and partition is very efficient through the fuzzy hypergraphs. Gong and Wang [24] represented the connection of fuzzy hypergraph with the fuzzy information system and the fuzzy concept lattice. Parvathi et al. [25] originated the notion of IF hypergraphs. Later on, this idea was extended by Akram and Dudek [26]. Akram and Luqman [27,28] introduced the intuitionistic single-valued neutrosophic and bipolar neutrosophic hypergraphs. Recently, $q$-ROF hypergraphs with applications were studied by Luqman et al. [29]. Luqman et al. [30] also introduced an $m$-polar fuzzy hypergraph model of GrC.

The fundamental goal of this research work is to introduce the $q$-rung picture fuzzy hypergraphs and to construct a hypergraph model of GrC possessing the characteristics of PFSs, as well as $q$-ROFSs. In the proposed model, the vertex of the $q$-rung picture fuzzy hypergraph denotes an object, and a $q$-rung picture fuzzy hyperedge represents a granule. By applying the most effective and flexible theory of $q$-RPFSs to hypergraphs, we not only broaden the space of uncertain and vague data, but also deal with neutral relationships between granules. Our proposed model of $\mathrm{GrC}$, based on $q$-rung picture fuzzy hypergraphs, is much closer to and considerable of human reasoning as compared to earlier concepts. In this model, the range of indication of decision data can be changed by changing the value of parameter $q, q \geq 1$. The rest of the paper is arranged as follows: In Section 2, $q$-rung picture fuzzy hypergraphs are defined. A $q$-rung picture fuzzy hierarchical quotient space structure is developed based on the $q$-rung picture fuzzy equivalence relation. In Section 3, we construct a $q$-rung picture fuzzy hypergraph model of $\mathrm{GrC}$. The partition and covering of granules are defined in the same section. The method of bottom-up construction is explained by an algorithm and an example. In Section 4, a model of $\mathrm{GrC}$ is constructed using level hypergraphs based on the $q$-rung picture fuzzy equivalence relation, and the construction of the level hypergraph model is illustrated through a concrete example. The "refinement" and "coarsening" operators are defined and their certain properties are discussed briefly. In Section 5, we compare the flexibility of our proposed model with existing models of $q$-ROFSs and PFSs. Section 6 deals with some concluding remarks and future studies.

\section{2. $q$-Rung Picture Fuzzy Hierarchical Structure}

Definition 1. [14] A q-rung orthopair fuzzy set ( $q$-ROFS) $Q$ in the universe $X$ is an object of the form,

$$
Q=\left\{\left(a, T_{Q}(a), F_{Q}(a)\right) \mid a \in X\right\},
$$

where $T_{Q}: X \rightarrow[0,1]$ represents the positive degree and $F_{Q}: X \rightarrow[0,1]$ represents the negative degree of the element $a \in X$ such that,

$$
0 \leq T_{Q}^{q}(a)+F_{Q}^{q}(a) \leq 1, q \geq 1, \text { for all } a \in X
$$
set $Q$.

Furthermore, $\pi_{Q}(a)=\sqrt[q]{1-T_{Q}^{q}(a)-F_{Q}^{q}(a)}$ is called a $q$-ROF index or indeterminacy degree of $a$ to the

Definition 2. [16] A picture fuzzy set (PFS) P is an object of the form,

$$
P=\left\{\left(a, T_{P}(a), N_{P}(a), F_{P}(a)\right) \mid a \in X\right\},
$$


where the mapping $T_{P}: X \rightarrow[0,1]$ represents the positive degree, $N_{P}: X \rightarrow[0,1]$ represents the neutral degree, and $F_{P}: X \rightarrow[0,1]$ represents the negative degree of the element $a \in X$ such that,

$$
0 \leq T_{P}(a)+N_{P}(a)+F_{P}(a) \leq 1, \text { for all } a \in X .
$$

The refusal degree of $a$ in $P$ is defined as $\left(1-T_{P}(a)-N_{P}(a)-F_{P}(a)\right)$.

Definition 3. [17] A q-rung picture fuzzy set (q-RPFS) $R$ is an object having the form,

$$
R=\left\{\left(a, T_{R}(a), N_{R}(a), F_{R}(a)\right) \mid a \in X\right\},
$$

where the function $T_{R}: X \rightarrow[0,1]$ represents the positive degree, $N_{R}: X \rightarrow[0,1]$ represents the neutral degree, and $F_{R}: X \rightarrow[0,1]$ represents the negative degree of the element $a \in X$ such that,

$$
0 \leq T_{R}^{q}(a)+N_{R}^{q}(a)+F_{R}^{q}(a) \leq 1, q \geq 1 \text {, for all } a \in X .
$$

The refusal degree of $a$ in $R$ is defined as $\sqrt[q]{\left(1-T_{P}^{q}(a)-N_{P}^{q}(a)-F_{P}^{q}(a)\right)}$.

Definition 4. The support of a $q$-RPFS $R=\left\{\left(a, T_{R}(a), N_{R}(a), F_{R}(a)\right) \mid a \in X\right\}$ is defined as,

$$
\operatorname{supp}(R)=\left\{a \mid T_{R}(a) \neq 0, N_{R}(a) \neq 1, F_{R}(a) \neq 1\right\} .
$$

The height of a $q$-RPFS $R=\left\{\left(a, T_{R}(a), N_{R}(a), F_{R}(a)\right) \mid a \in X\right\}$ is defined as,

$$
h(R)=\left\{\left(\max \left\{T_{R}(a)\right\}, \min \left\{N_{R}(a)\right\}, \min \left\{F_{R}(a)\right\}\right) \mid a \in X\right\} .
$$

Definition 5. Let $M$ and $L$ be two $q$-RPFSs on $X$. The union and intersection of $M$ and $L$ are defined as,

(i) $\quad M \subseteq L \Leftrightarrow T_{M}(x) \leq T_{L}(x), N_{M}(x) \geq N_{L}(x), F_{M}(x) \geq F_{L}(x)$.

(ii) $M \cup L=\left\{\left(x, \max \left(T_{M}(x), T_{L}(x)\right), \min \left(N_{M}(x), N_{L}(x)\right), \min \left(F_{M}(x), F_{L}(x)\right)\right) \mid x \in X\right\}$.

(iii) $M \cap L=\left\{\left(x, \min \left(T_{M}(x), T_{L}(x)\right), \min \left(N_{M}(x), N_{L}(x)\right), \max \left(F_{M}(x), F_{L}(x)\right)\right) \mid x \in X\right\}$.

Definition 6. Let $X$ be a set of universes. A q-rung picture fuzzy hypergraph ( $q-R P F H$ ) on $X$ is defined as an ordered pair $H=(\mathcal{R}, S)$, where $\mathcal{R}=\left\{R_{1}, R_{2}, R_{3}, \ldots, R_{l}\right\}$ is a collection of non-trivial $q$-RPFSs on $X$ and $S$ is a $q$-RPFR on $R_{i}$ such that:

1. $T_{S}\left(E_{l}\right)=T_{S}\left(\left\{r_{1}, r_{2}, r_{3}, \cdots, r_{k}\right\}\right) \leq \min \left\{T_{R_{i}}\left(r_{1}\right), T_{R_{i}}\left(r_{2}\right), T_{R_{i}}\left(r_{3}\right), \cdots, T_{R_{i}}\left(r_{k}\right)\right\}$,

$N_{S}\left(E_{l}\right)=N_{S}\left(\left\{r_{1}, r_{2}, r_{3}, \cdots, r_{k}\right\}\right) \leq \min \left\{N_{R_{i}}\left(r_{1}\right), N_{R_{i}}\left(r_{2}\right), N_{R_{i}}\left(r_{3}\right), \cdots, N_{R_{i}}\left(r_{k}\right)\right\}$,

$F_{S}\left(E_{l}\right)=F_{S}\left(\left\{r_{1}, r_{2}, r_{3}, \cdots, r_{k}\right\}\right) \leq \max \left\{F_{R_{i}}\left(r_{1}\right), F_{R_{i}}\left(r_{2}\right), F_{R_{i}}\left(r_{3}\right), \cdots, F_{R_{i}}\left(r_{k}\right)\right\}$,

for all $r_{1}, r_{2}, r_{3}, \cdots, r_{k} \in X$.

2. $X=\bigcup_{k} \operatorname{supp}\left(R_{i}\right)$, for all $R_{i} \in \mathcal{R}$.

Here, $E=\left\{E_{1}, E_{2}, E_{3}, \cdots, E_{l}\right\}$ is the family of hyperedges.

Definition 7. $A q-R P F H H=(\mathcal{R}, S)$ is simple if $H$ does not contain repeated $q-R P F$ hyperedges, and whenever $E_{i} \subseteq E_{j}, E_{i}, E_{j} \in E$, then $E_{i}=E_{j}$.

Definition 8. Let $H=(\mathcal{R}, S)$ be a $q$-RPFH and $E_{i} \in$ E. Suppose $\alpha, \beta, \gamma \in[0,1]$; the $(\alpha, \beta, \gamma)$-cut of $E_{i}$, $E_{i}^{(\alpha, \beta, \gamma)}$, is given as:

$$
E_{i}^{(\alpha, \beta, \gamma)}=\left\{a \in X \mid T_{E_{i}}(a) \geq \alpha, N_{E_{i}}(a) \geq \beta, F_{E_{i}}(a) \leq \gamma\right\}
$$

Let:

- $E^{(\alpha, \beta, \gamma)}=\left\{E_{i}^{(\alpha, \beta, \gamma)} \mid E_{i} \in E\right\}$,

- $X^{(\alpha, \beta, \gamma)}=\cup\left\{E_{i}^{(\alpha, \beta, \gamma)} \mid E_{i} \in E\right\}$. 
If $E^{(\alpha, \beta, \gamma)}$ is non-empty, then the $(\alpha, \beta, \gamma)$-level hypergraph of $H$ is defined as $H^{(\alpha, \beta, \gamma)}=\left(X^{(\alpha, \beta, \gamma)}\right.$, $\left.E^{(\alpha, \beta, \gamma)}\right)$, which is a crisp hypergraph.

We now discuss the $q$-rung picture fuzzy hierarchical quotient space structure.

Different techniques have been proposed to deal with GrC. Quotient space (QS), FSs, and rough sets are three basic computing tools dealing with uncertainty. Based on FSs, the fuzzy equivalence relation (FER), as an extension of equivalence relation, was proposed by Zadeh [31]. The question of distinct membership degrees of the same object from different scholars has arisen because of various ways of thinking about the interpretation of different functions dealing with the same problem. To resolve this issue, FS was structurally defined by Zhang and Zhang [32], which was based on QS theory and FER [33]. This definition provides some new intuitiveness regarding the membership degree, called a hierarchical QS structure of an FER. By following the same concept, we develop a hierarchical QS structure of a $q$-RPFER.

Definition 9. Let $X_{1}$ and $X_{2}$ be two finite sets, then the Cartesian product between $X_{1}$ and $X_{2}$ is $X_{1} \times X_{2}$. Every $q-R P F$ subset $R$ of $X_{1} \times X_{2}$ is defined as a q-RPF binary relation from $X_{1}-X_{2}$. Let $X_{1}=$ $\left\{r_{1}, r_{2}, r_{3}, \cdots, r_{l}\right\}$ and $X_{2}=\left\{s_{1}, s_{2}, s_{3}, \cdots, s_{m}\right\}$, a $q$-RPF binary relation matrix $\tilde{M}_{R}$, be given as follows,

$$
\tilde{M}_{R}=\left[\begin{array}{cccc}
d_{R}\left(r_{1}, s_{1}\right) & d_{R}\left(r_{1}, s_{2}\right) & \cdots & d_{R}\left(r_{1}, s_{m}\right) \\
d_{R}\left(r_{2}, s_{1}\right) & d_{R}\left(r_{2}, s_{2}\right) & \cdots & d_{R}\left(r_{2}, s_{m}\right) \\
\vdots & \vdots & \vdots & \vdots \\
d_{R}\left(r_{l}, s_{1}\right) & d_{R}\left(r_{l}, s_{2}\right) & \cdots & d_{R}\left(r_{l}, s_{m}\right)
\end{array}\right] .
$$

In general, $\tilde{M}_{R}$ is called $q$-RPF relation matrix of $R$, where $d_{R}(r, s)=\left(T_{R}(r, s), N_{R}(r, s), F_{R}(r, s)\right)$, and $T_{R}: X_{1} \times X_{2} \rightarrow[0,1], N_{R}: X_{1} \times X_{2} \rightarrow[0,1], F_{R}: X_{1} \times X_{2} \rightarrow[0,1]$ represent the positive degree, neutral degree, and negative degree of $r$ and $s$, respectively, such that:

$$
0 \leq T_{R}^{q}(r, s)+N_{R}^{q}(r, s)+F_{R}^{q}(r, s) \leq 1, q \geq 1,
$$

for all $(r, s) \in X_{1} \times X_{2}$.

Definition 10. A q-RPFR is called a $q-R P F$ similarity relation if it satisfies,

1. $d_{R}(r, r)=(1,0,0)$, for all $r \in X$,

2. $d_{R}(r, s)=d_{R}(s, r)$, for all $r, s \in X$.

Definition 11. A q-RPFR is called a q-RPF equivalence relation ( $q$-RPFER) if the following conditions are satisfied,

1. $d_{R}(r, r)=(1,0,0)$, for all $r \in X$,

2. $d_{R}(r, s)=d_{R}(s, r)$, for all $r, s \in X$,

3. for all $r, s, t \in X$

(i) $T_{R}(r, t)=\sup _{s \in X}\left\{\min \left(T_{R}(r, s), T_{R}(s, t)\right)\right\}$,

(ii) $N_{R}(r, t)=\sup \left\{\min \left(N_{R}(r, s), N_{R}(s, t)\right)\right\}$,

(iii) $F_{R}(r, t)=\inf _{s \in X}\left\{\max \left(F_{R}(r, s), F_{R}(s, t)\right)\right\}$.

A $q$-RPF quotient space ( $q$-RPFQS) is denoted by a triplet $(X, \tilde{A}, R)$, where $X$ is a finite domain, $\tilde{A}$ represents the attributes of $X$, and $R$ represents the $q$-RPF relationship between the objects of universe $X$, which is called the structure of the domain. 
Definition 12. Let $x_{i}$ and $x_{j}$ be two objects in the universe $X$. The similarity between $x_{i}, x_{j} \in X$ having the attribute $\tilde{a}_{k}$ is defined as:

$$
T_{R}\left(x_{i}, x_{j}\right)=\frac{\left|\tilde{a}_{i k} \cap \tilde{a}_{j k}\right|}{\left|\tilde{a}_{i k} \cup \tilde{a}_{j k}\right|}, N_{R}\left(x_{i}, x_{j}\right)=\frac{\left|\tilde{a}_{i k} \cap \tilde{a}_{j k}\right|}{\left|\tilde{a}_{i k} \cup \tilde{a}_{j k}\right|}, F_{R}\left(x_{i}, x_{j}\right)=\frac{\left|\tilde{a}_{i k} \cup \tilde{a}_{j k}\right|}{\left|\tilde{a}_{i k} \cap \tilde{a}_{j k}\right|},
$$

where $\tilde{a}_{i k}$ represents that object $x_{i}$ possesses the attribute $\tilde{a}_{k}$ and $\tilde{a}_{j k}$ represents that object $x_{j}$ possesses the attribute $\tilde{a}_{k}$.

It is noted that the $q$-RPFR matrix $\tilde{M}_{R}$ is symmetric and reflexive $q$-RPFR, but in general, it does not satisfy the transitivity condition.

Proposition 1. Let $R$ be a $q$-RPFR on a finite domain $X$ and $R^{(\alpha, \beta, \gamma)}=\left\{(x, y) \mid T_{R}(x, y) \geq \alpha, N_{R}(x, y) \geq\right.$ $\left.\beta, F_{R}(x, y) \leq \gamma\right\},(\alpha, \beta, \gamma) \in[0,1]$. Then, $R^{(\alpha, \beta, \gamma)}$ is an ER on $X$ and is said to be a cut-equivalence relation of $R$.

Proposition 1 depicts that $R^{(\alpha, \beta, \gamma)}$ is a crisp relation, which is an equivalence relation on $X$, and its knowledge space is given as $\tau_{R^{(\alpha, \beta, \gamma)}}(X)=X / R^{(\alpha, \beta, \gamma)}$.

The value domainof an ER $R$ on $X$ is defined as $D=\left\{d_{R}(x, y) \mid x, y \in X\right\}$ such that,

(i) $T_{X}(x) \wedge T_{X}(y) \wedge T_{R}(x, y)>0$,

(ii) $N_{X}(x) \wedge N_{X}(y) \wedge N_{R}(x, y)>0$,

(iii) $F_{X}(x) \vee F_{X}(y) \vee F_{R}(x, y)>0$.

Definition 13. Let $R$ be a $q$-RPFER on a finite set $X$. The set given by $\tau_{X}(R)=\left\{X / R^{(\alpha, \beta, \gamma)} \mid(\alpha, \beta, \gamma) \in D\right\}$ is called the $q$-RPF hierarchical quotient space structure ( $q$-RPFHQSS) of $R$, where $D$ is the value domain.

Example 1. Let $X=\left\{s_{1}, s_{2}, s_{3}, s_{4}, s_{5}\right\}$ and $R$ be a 5 -RPFER on $X$; the corresponding relation matrix $\tilde{M}_{R}$ is given as follows,

$$
\tilde{M}_{R}=\left[\begin{array}{ccccc}
(1,0,0) & (0.3,0.4,0.3) & (0.7,0.5,0.1) & (0.5,0.5,0.2) & (0.5,0.5,0.2) \\
(0.3,0.4,0.3) & (1,0,0) & (0.3,0.4,0.3) & (0.3,0.4,0.3) & (0.3,0.4,0.3) \\
(0.7,0.5,0.1) & (0.3,0.4,0.3) & (1,0,0) & (0.5,0.5,0.2) & (0.5,0.5,0.2) \\
(0.5,0.5,0.2) & (0.3,0.4,0.3) & (0.5,0.5,0.2) & (1,0,0) & (0.9,0.5,0.1) \\
(0.5,0.5,0.2) & (0.3,0.4,0.3) & (0.5,0.5,0.2) & (0.9,0.5,0.1) & (1,0,0)
\end{array}\right]
$$

Its corresponding HQSS is given as,

$$
\begin{aligned}
& X / R^{\left(\alpha_{1}, \beta_{1}, \gamma_{1}\right)}=\left\{\left\{s_{1}, s_{2}, s_{3}, s_{4}, s_{5}\right\}\right\}, 0<\alpha_{1} \leq 0.3,0<\beta_{1} \leq 0.4,0.3>\gamma_{1} \geq 0, \\
& X / R^{\left(\alpha_{2}, \beta_{2}, \gamma_{2}\right)}=\left\{\left\{s_{2}\right\},\left\{s_{1}, s_{3}, s_{4}, s_{5}\right\}\right\}, 0.3<\alpha_{2} \leq 0.5,0.4<\beta_{2} \leq 0.5,0.3>\gamma_{2} \geq 0.2, \\
& X / R^{\left(\alpha_{3}, \beta_{3}, \gamma_{3}\right)}=\left\{\left\{s_{1}, s_{3}\right\},\left\{s_{2}\right\},\left\{s_{4}, s_{5}\right\}\right\}, 0.5<\alpha_{3} \leq 0.7,0.5<\beta_{3} \leq 0.5,0.2>\gamma_{3} \geq 0.1, \\
& X / R^{\left(\alpha_{4}, \beta_{4}, \gamma_{4}\right)}=\left\{\left\{s_{1}\right\},\left\{s_{2}\right\},\left\{s_{3}\right\},\left\{s_{4}, s_{5}\right\}\right\}, 0.7<\alpha_{4} \leq 0.9,0.5<\beta_{4} \leq 0.5,0.1>\gamma_{4} \geq 0.1, \\
& X / R^{\left(\alpha_{5}, \beta_{5}, \gamma_{5}\right)}=\left\{\left\{s_{1}\right\},\left\{s_{2}\right\},\left\{s_{3}\right\},\left\{s_{4}\right\},\left\{s_{5}\right\}\right\}, 0.9<\alpha_{5} \leq 1,0.5<\beta_{5} \leq 1,0.1>\gamma_{4} \geq 0 .
\end{aligned}
$$

Hence, a HQSS induced by 5-RPFER $R$ is given as $\tau_{X}(R)=$ $\left\{X / R^{\left(\alpha_{1}, \beta_{1}, \gamma_{1}\right)}, X / R^{\left(\alpha_{2}, \beta_{2}, \gamma_{2}\right)}, X / R^{\left(\alpha_{3}, \beta_{3}, \gamma_{3}\right)}, X / R^{\left(\alpha_{4}, \beta_{4}, \gamma_{4}\right)}, X / R^{\left(\alpha_{5}, \beta_{5}, \gamma_{5}\right)}\right\}$ and is shown in Figure 1. 


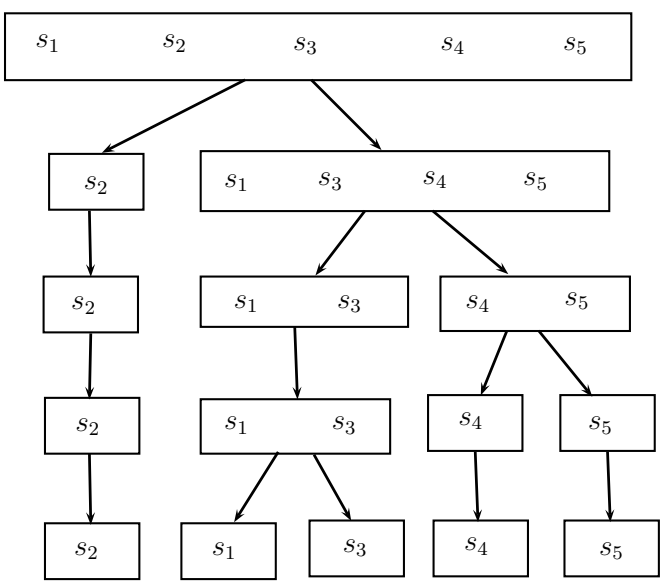

Figure 1. The hierarchical quotient space structure (HQSS) of $R$.

Furthermore, assuming the number of blocks in every distinct layer of this HQSS, a pyramid model can also be constructed as shown in Figure 2.

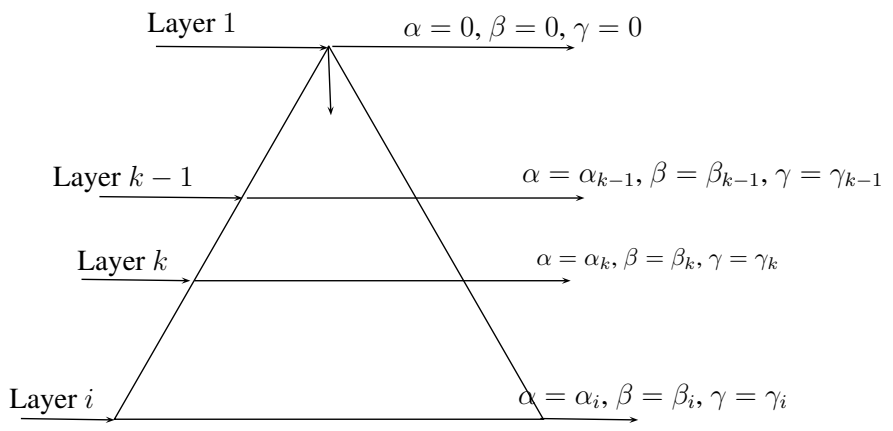

Figure 2. The pyramid model of an HQSS.

Note that the same HQSS can be formed by different 5-rung picture fuzzy equivalence relations. For instance, the relation matrix $\tilde{M}_{R_{1}}$ of 5-RPFER generates the same HQSS as given by $\tilde{M}_{R}$. The relation matrix $\tilde{M}_{R_{1}}$ is given as,

$$
\tilde{M}_{R_{1}}=\left[\begin{array}{ccccc}
(1,0,0) & (0.2,0.3,0.2) & (0.9,0.5,0.1) & (0.6,0.6,0.3) & (0.6,0.6,0.3) \\
(0.2,0.3,0.2) & (1,0,0) & (0.2,0.3,0.2) & (0.2,0.3,0.2) & (0.2,0.3,0.2) \\
(0.9,0.5,0.1) & (0.2,0.3,0.2) & (1,0,0) & (0.6,0.6,0.3) & (0.6,0.6,0.3) \\
(0.6,0.6,0.3) & (0.2,0.3,0.2) & (0.6,0.6,0.3) & (1,0,0) & (0.7,0.5,0.1) \\
(0.6,0.6,0.3) & (0.2,0.3,0.2) & (0.6,0.6,0.3) & (0.7,0.5,0.1) & (1,0,0)
\end{array}\right]
$$

Definition 14. Let $R$ be a q-RPFER on $X$. Let $\tau_{X}(R)=\left\{X\left(\rho_{1}\right), X\left(\rho_{2}\right), X\left(\rho_{3}\right), \cdots\right.$, and $\left.X\left(\rho_{j}\right)\right\}$ be its corresponding HQSS, where $\rho_{i}=\left(\alpha_{i}, \beta_{i}, \gamma_{i}\right), i=1,2, \cdots, j$ and $X\left(\rho_{j}\right)<X\left(\rho_{j-1}\right)<\cdots<X\left(\rho_{1}\right)$. Then, the partition sequence of $\tau_{X}(R)$ is given as $P\left(\tau_{X}(R)\right)=\left\{P_{1}, P_{2}, P_{3}, \cdots, P_{j}\right\}$, where $P_{i}=\left|X\left(\rho_{i}\right)\right|$, $i=1,2, \cdots, j$, and $|$.$| represents the total number of elements in a set.$

Definition 15. Let $\tau_{X}(R)=\left\{X\left(\rho_{1}\right), X\left(\rho_{2}\right), X\left(\rho_{3}\right), \cdots, X\left(\rho_{j}\right)\right\}$ be its corresponding HQSS, where $\rho_{i}=$ $\left(\alpha_{i}, \beta_{i}, \gamma_{i}\right), i=1,2, \cdots, j$ and $X\left(\rho_{j}\right)<X\left(\rho_{j-1}\right)<\cdots<X\left(\rho_{1}\right), P\left(\tau_{X}(R)\right)=\left\{P_{1}, P_{2}, P_{3}, \cdots, P_{j}\right\}$ be the partition sequence of $\tau_{X}(R)$. Assume that $X\left(\rho_{i}\right)=\left\{X_{i 1}, X_{i 2}, \cdots, X_{i P_{i}}\right\}$. The information entropy $I_{X}\left(\rho_{i}\right)$ is defined as $I_{X}\left(\rho_{i}\right)=-\sum_{t=1}^{P_{i}} \frac{\left|X_{i t}\right|}{|X|} \ln \left(\frac{\left|X_{i t}\right|}{|X|}\right)$. 
Theorem 1. Let $R$ be a q-RPFER on $X$. Let $\tau_{X}(R)=\left\{X\left(\rho_{1}\right), X\left(\rho_{2}\right), X\left(\rho_{3}\right), \cdots, X\left(\rho_{j}\right)\right\}$ be its corresponding HQSS, where $\rho_{i}=\left(\alpha_{i}, \beta_{i}, \gamma_{i}\right), i=1,2, \cdots, j$, then the entropy sequence $I\left(\tau_{X}(R)\right)=$ $\left\{I_{X}\left(\rho_{1}\right), I_{X}\left(\rho_{2}\right), \cdots, I_{X}\left(\rho_{j}\right)\right\}$ increases monotonically and strictly.

Proof. The terminology of HQSS implies that $X\left(\rho_{j}\right)<X\left(\rho_{j-1}\right)<\cdots<X\left(\rho_{1}\right)$, i.e., $X\left(\rho_{j-1}\right)$ is a quotient subspace of $X\left(\rho_{j}\right)$. Suppose that $X\left(\rho_{i}\right)=\left\{X_{i 1}, X_{i 2}, \cdots, X_{i P_{i}}\right\}$ and $X\left(\rho_{i-1}\right)=$ $\left\{X_{(i-1) 1}, X_{(i-1) 2}, \cdots, X_{(i-1) P_{(i-1)}}\right\}$, then every sub-block of $X\left(\rho_{i-1}\right)$ is an amalgam of sub-blocks of $X\left(\rho_{i}\right)$. Without loss of generality, it is assumed that only one sub-block $X_{i-1, j}$ in $X\left(\rho_{i-1}\right)$ is formed by the combination of two sub-blocks $X_{i r}, X_{i s}$ in $X\left(\rho_{i}\right)$, and all other remaining blocks are equal in both sequences. Thus,

$$
\begin{aligned}
I_{X}\left(\rho_{j-1}\right) & =-\sum_{t=1}^{P_{i-1}} \frac{\left|X_{i-1, t}\right|}{|X|} \ln \left(\frac{\left|X_{i-1, t}\right|}{|X|}\right) \\
& =-\sum_{t=1}^{P_{j-1}} \frac{\left|X_{i-1, t}\right|}{|X|} \ln \left(\frac{\left|X_{i-1, t}\right|}{|X|}\right)-\sum_{t=j+1}^{P_{i-1}} \frac{\left|X_{i-1, t}\right|}{|X|} \ln \left(\frac{\left|X_{i-1, t}\right|}{|X|}\right)-\frac{\left|X_{i-1, j}\right|}{|X|} \ln \left(\frac{\left|X_{i-1, j}\right|}{|X|}\right) \\
& =-\sum_{t=1}^{P_{j-1}} \frac{\left|X_{i, t}\right|}{|X|} \ln \left(\frac{\left|X_{i, t}\right|}{|X|}\right)-\sum_{t=j+1}^{P_{i}} \frac{\left|X_{i, t}\right|}{|X|} \ln \left(\frac{\left|X_{i, t}\right|}{|X|}\right)-\frac{\left|X_{i, r}\right|+\left|X_{i, s}\right|}{|X|} \ln \left(\frac{\left|X_{i, r}\right|+\left|X_{i, s}\right|}{|X|}\right) .
\end{aligned}
$$

Since,

$$
\begin{aligned}
\frac{\left|X_{i, r}\right|+\left|X_{i, s}\right|}{|X|} \ln \left(\frac{\left|X_{i, r}\right|+\left|X_{i, s}\right|}{|X|}\right) & =\frac{\left|X_{i, r}\right|}{|X|} \ln \left(\frac{\left|X_{i, r}\right|+\left|X_{i, s}\right|}{|X|}\right)+\frac{\left|X_{i, s}\right|}{|X|} \ln \left(\frac{\left|X_{i, r}\right|+\left|X_{i, s}\right|}{|X|}\right) \\
& >\frac{\left|X_{i, r}\right|}{|X|} \ln \left(\frac{\left|X_{i, r}\right|}{|X|}\right)+\frac{\left|X_{i, s}\right|}{|X|} \ln \left(\frac{\left|X_{i, s}\right|}{|X|}\right) .
\end{aligned}
$$

Therefore, we have:

$$
\begin{aligned}
I_{X}\left(\rho_{j-1}\right) & <-\sum_{t=1}^{P_{j-1}} \frac{\left|X_{i, t}\right|}{|X|} \ln \left(\frac{\left|X_{i, t}\right|}{|X|}\right)-\sum_{t=j+1}^{P_{i}} \frac{\left|X_{i, t}\right|}{|X|} \ln \left(\frac{\left|X_{i, t}\right|}{|X|}\right)-\frac{\left|X_{i, r}\right|}{|X|} \ln \left(\frac{\left|X_{i, r}\right|}{|X|}\right)-\frac{\left|X_{i, s}\right|}{|X|} \ln \left(\frac{\left|X_{i, s}\right|}{|X|}\right) \\
& =I_{X}\left(\rho_{j}\right),(2 \leq j \leq n) .
\end{aligned}
$$

Hence, $I_{X}\left(\rho_{1}\right)<I_{X}\left(\rho_{2}\right)<I_{X}\left(\rho_{2}\right)<\cdots<I_{X}\left(\rho_{j}\right)$.

Definition 16. Let $X=\left\{x_{1}, x_{2}, x_{3}, \cdots, x_{n}\right\}$ be a non-empty set of universes, and let $\mathcal{P}_{t}(X)=$ $\left\{X_{1}, X_{2}, X_{3}, \cdots, X_{t}\right\}$ be a partition space (PS) of $X$, where $\left|\mathcal{P}_{t}(X)\right|=t$, then $\mathcal{P}_{t}(X)$ is called the $t$-order partition space ( $t$-OPS) on $X$.

Definition 17. Let $\mathcal{P}_{t}(X)=\left\{X_{1}, X_{2}, X_{3}, \cdots, X_{t}\right\}$ be a $t$-OPS on $X$. Let $\left|X_{1}\right|=b_{1},\left|X_{2}\right|=b_{2}, \cdots$, $\left|X_{t}\right|=b_{t}$, and the sequence $\left\{b_{1}, b_{2}, \cdots, b_{t}\right\}$ is arranged in increasing order, then we get a new sequence $H(t)=\left\{b_{1}^{\prime}, b_{2}^{\prime}, \cdots, b_{t}^{\prime}\right\}$, which is also increasing and called a sub-block sequence of $\mathcal{P}_{t}(X)$.

Note that two different $t$-order partition spaces on $X$ may possess the similar sub-block sequence $H(t)$.

Definition 18. Let $\mathcal{P}_{t}(X)=\left\{X_{1}, X_{2}, X_{3}, \cdots, X_{t}\right\}$ be a partition space of $X$. Suppose that $H_{1}(t)=$ $\left\{b_{1}^{\prime}, b_{2}^{\prime}, \cdots, b_{t}^{\prime}\right\}$ is a sub-block sequence of $\mathcal{P}_{t}(X)$, then the $\omega$-displacement of $H_{1}(t)$ is defined as as the increasing sequence $H_{2}(t)=\left\{b_{1}^{\prime}, b_{2}^{\prime}, \cdots, b_{r}^{\prime}+1, \cdots, b_{s}^{\prime}-1, \cdots, b_{t}^{\prime}\right\}$, where $r<s, b_{r}^{\prime}+1<b_{s}^{\prime}-1$.

An $\omega$-displacement is obtained by subtracting one from some bigger term and adding one to the smaller one such that the sequence does not change its increasing property. 
Theorem 2. A single time $\omega$-displacement $H_{2}(t)$, derived from $H_{1}(t)$, satisfies $I\left(H_{1}(t)\right)<I\left(H_{2}(t)\right)$.

Proof. Let $H_{1}(t)=\left\{p_{1}^{\prime}, p_{2}^{\prime}, \cdots, p_{t}^{\prime}\right\}$, and $H_{2}(t)=\left\{p_{1}^{\prime}, p_{2}^{\prime}, \cdots, p_{r}^{\prime}+1, \cdots, p_{s}^{\prime}-1, \cdots, p_{t}^{\prime}\right\}, p_{1}^{\prime}+p_{2}^{\prime}+$ $\cdots+p_{t}^{\prime}=q$, then we have,

$$
I\left(H_{2}(t)\right)=-\sum_{j=1}^{t} \frac{p_{l}^{\prime}}{q} \ln \frac{p_{l}^{\prime}}{q}+\frac{p_{r}^{\prime}}{q} \ln \frac{p_{r}^{\prime}}{q}+\frac{p_{s}^{\prime}}{q} \ln \frac{p_{s}^{\prime}}{q}-\frac{p_{r}^{\prime}+1}{q} \ln \frac{p_{r}^{\prime}+1}{q}-\frac{p_{s}^{\prime}-1}{q} \ln \frac{p_{s}^{\prime}-1}{q} .
$$

Let $\varphi(x)=-\frac{x}{q} \ln \frac{x}{q}-\frac{l-x}{q} \ln \frac{l-x}{q}$, where $l=p_{r}^{\prime}+p_{s}^{\prime}$ and $\varphi^{\prime}(x)=\frac{1}{q} \ln \frac{l-x}{x}$. Suppose that $\varphi^{\prime}(x)=0$, then we obtain a solution, i.e., $x=\frac{l}{2}$. Furthermore, $\varphi^{\prime \prime}(x)=\frac{-l}{q(l-x) x}<0,0 \leq x \leq \frac{l}{2}$, and $\varphi(x)$ is increasing monotonically. Let $x_{1}=p_{r}^{\prime}$ and $x_{2}=p_{r}^{\prime}+1, p_{r}^{\prime}+1<p_{s}^{\prime}-1$, i.e., $x_{1}<x_{2} \leq \frac{l}{2}=\frac{p_{r}^{\prime}+p_{s}^{\prime}}{2}$. Since, $\varphi(x)$ is monotone, then $\varphi\left(x_{2}\right)-\varphi\left(x_{1}\right)>0$. Thus,

$$
\frac{p_{r}^{\prime}}{q} \ln \frac{p_{r}^{\prime}}{q}+\frac{p_{s}^{\prime}}{q} \ln \frac{p_{s}^{\prime}}{q}-\frac{p_{r}^{\prime}+1}{q} \ln \frac{p_{r}^{\prime}+1}{q}-\frac{p_{s}^{\prime}-1}{q} \ln \frac{p_{s}^{\prime}-1}{q}>0
$$

Therefore, we have:

$$
\begin{aligned}
I\left(H_{2}(t)\right) & =-\sum_{j=1}^{t} \frac{p_{l}^{\prime}}{q} \ln \frac{p_{l}^{\prime}}{q}+\frac{p_{r}^{\prime}}{q} \ln \frac{p_{r}^{\prime}}{q}+\frac{p_{s}^{\prime}}{q} \ln \frac{p_{s}^{\prime}}{q}-\frac{p_{r}^{\prime}+1}{q} \ln \frac{p_{r}^{\prime}+1}{q}-\frac{p_{s}^{\prime}-1}{q} \ln \frac{p_{s}^{\prime}-1}{q} \\
& >-\left(\frac{p_{r}^{\prime}+1}{q} \ln \frac{p_{r}^{\prime}+1}{q}+\frac{p_{s}^{\prime}-1}{q} \ln \frac{p_{s}^{\prime}-1}{q}\right) \\
& >-\sum_{j=1}^{t} \frac{p_{l}^{\prime}}{q} \ln \frac{p_{l}^{\prime}}{q} \\
& =I\left(H_{1}(t)\right) .
\end{aligned}
$$

\section{Formation of the Model of GrC}

\subsection{Construction of Model}

GrC may utilize frameworks in terms of levels and granular structures, which are built on the basis of multiple representations and multiple levels. A granule is defined as a group of elements having the same attributes or properties and are taken as a whole.

Definition 19. A system $(X, R)$ is called an object space, where $X$ is a universe of objects or elements and $R=\left\{r_{1}, r_{2}, r_{3}, \cdots, r_{k}\right\}, k=|X|$ is a family of relations between the elements of $X$. For $n \leq k, r_{n} \in R$, $r_{n} \subseteq X \times X \times X \cdots \times X$, if $\left(x_{1}, x_{2}, \cdots, x_{n}\right) \subseteq r_{n}$, then there exists an n-array relation $r_{n}$ on $\left(x_{1}, x_{2}, \cdots, x_{n}\right)$.

The elements of an object space having some relation $r_{i} \in R$ can be assumed as a granule. A single object is considered as the smallest granule, and the set of all elements is said to be the largest granule in an object space.

We consider one vertex of a $q$-RPFH as a representation of an object, and the group of elements having some relationship $s_{i}$ is represented by the $q$-RPF hyperedge. The positive membership $T$ of an element $x_{i}$ refers to the belonging; neutral membership $N$ refers to the unbiased behavior; and negative membership $F$ refers to the disconnection to the granule, where $T\left(x_{i}\right), N\left(x_{i}\right), F\left(x_{i}\right) \in[0,1]$, satisfying the condition $T^{q}\left(x_{i}\right)+N^{q}\left(x_{i}\right)+F^{q}\left(x_{i}\right) \leq 1, q \geq 1$. Thus, we can establish a $q$-RPFH model of GrC. 
Example 2. Let $X=\left\{s_{1}, s_{2}, s_{3}, s_{4}, s_{5}\right\}$ be the set of objects and $R=\left\{r_{1}^{\prime}, r_{2}^{\prime}, r_{3}^{\prime}, r_{4}^{\prime}, r_{5}^{\prime}, r_{6}^{\prime}\right\}$ be the set of relations. A hypergraph $H=(\mathcal{R}, S)$ representing the objects and the relations $r_{i}^{\prime} \in R(1 \leq i \leq 6)$ between them is shown in Figure 3. By assigning the positive membership $T \in[0,1]$, neutral membership $N \in[0,1]$, and negative membership $F \in[0,1]$ to each element $s_{i}$, we form a seven-rung picture fuzzy hypergraph. Let $X=\left\{s_{1}, s_{2}, s_{3}, s_{4}, s_{5}\right\}$ and $S=\left\{S_{1}, S_{2}, S_{3}, S_{4}\right\}$, and the corresponding incidence matrix is given in Table 1 .

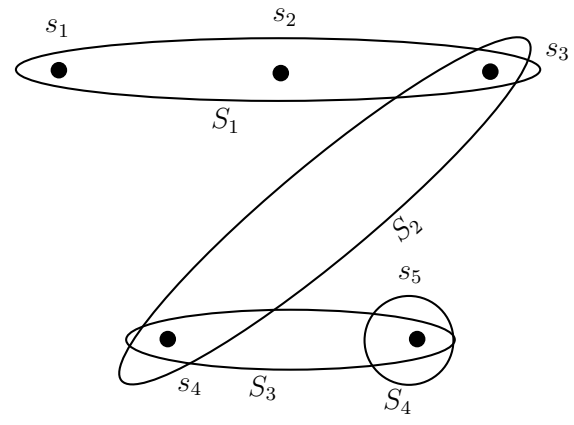

Figure 3. Hypergraph representation of granules.

Table 1. Incidence matrix of $H$.

\begin{tabular}{lllll}
\hline $\boldsymbol{I}$ & $\boldsymbol{S}_{\mathbf{1}}$ & $\boldsymbol{S}_{\mathbf{2}}$ & $\boldsymbol{S}_{\mathbf{3}}$ & $\boldsymbol{S}_{\boldsymbol{4}}$ \\
\hline$s_{1}$ & $(0.7,0.2,0.1)$ & $(0,0,1)$ & $(0,0,1)$ & $(0,0,1)$ \\
$s_{2}$ & $(0.8,0.2,0.3)$ & $(0,0,1)$ & $(0,0,1)$ & $(0,0,1)$ \\
$s_{3}$ & $(0.5,0.3,0.4)$ & $(0.5,0.3,0.1)$ & $(0,0,1)$ & $(0,0,1)$ \\
$s_{4}$ & $(0,0,1)$ & $(0.6,0.2,0.1)$ & $(0.5,0.3,0.1)$ & $(0,0,1)$ \\
$s_{5}$ & $(0,0,1)$ & $(0,0,1)$ & $(0.6,0.2,0.1)$ & $(0.5,0.3,0.1)$ \\
\hline
\end{tabular}

Let $\alpha=0.5, \beta=0.3, \gamma=0.5$, then we have $r_{1}^{\prime}=\left\{\left(s_{3}\right),\left(s_{4}\right)\right\}, r_{2}^{\prime}=\left\{\left(s_{2}, s_{4}\right)\right\}, r_{3}^{\prime}, r_{4}^{\prime}, r_{5}^{\prime}=\{\varnothing\}$. Hypergraph representation of granules in a level is given in Figure 4.

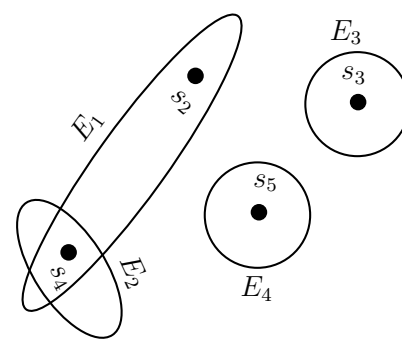

Figure 4. Representation of granules in a level through the hypergraph.

The relationships between the vertices can be obtained by following the present situation, through computing the mathematical function or by figuring out the internal, external, and contextual properties of the granule. After the relationship is computed, the set of vertices possessing the relationship among them can be combined into one module. By taking into account the actual condition, we can calculate or assign positive, neutral, and negative degrees through the membership functions $T(s), N(s)$, and $F(s)$, respectively. As a result, we have formed a granule that is also called the $q$-RPF hyperedge. The $(\alpha, \beta, \gamma)$-cuts are computed for this $q$-RPF hyperedge. When all vertices under consideration are to be integrated into a single unit and the membership degrees are assigned or computed for the unit, we have constructed a single-level model of GrC.

The amalgamation of the elements or objects that are processed at the same time while resolving a problem is called a granule. It reflects the representation of the perceptions and the attributes of the integration. A granule may be a small part of another granule and can also be considered as 
the block to form larger granules. It can also be a collection of granules or may be thought of as a whole unit. Thus, a granule may play two distinct roles.

Definition 20. A partition of a set X established on the basis of relations between objects is defined as a collection of non-empty subsets that are pairwise disjoint and whose union is the whole of $X$. These subsets that form the partition of $X$ are called blocks. Every partition of a finite set $X$ contains the finite number of blocks. Corresponding to the $q-R P F H$, the constraints of partition $\psi=\left\{\mathcal{E}_{i} \mid 1 \leq i \leq n\right\}$ can be stated as follows,

(i) every $\mathcal{E}_{i}$ is non-empty,

(ii) $\mathcal{E}_{i} \cap \mathcal{E}_{j}=\varnothing$, for $i \neq j$,

(iii) $\cup\left\{\operatorname{supp}\left(\mathcal{E}_{i}\right) \mid 1 \leq i \leq n\right\}=X$.

Definition 21. A covering $c=\left\{\mathcal{E}_{i} \mid 1 \leq i \leq n\right\}$ of a set $X$ is defined as non-empty subsets of $X$ such that,

(i) every $\mathcal{E}_{i}$ is non-trivial,

(ii) $\cup\left\{\operatorname{supp}\left(\mathcal{E}_{i}\right) \mid 1 \leq i \leq n\right\}=X$.

In a $q$-RPFH, if $S_{i}, S_{j} \in S$, and $S_{i} \cap S_{j}=\varnothing$, i.e., $S_{i}$ and $S_{j}$ do not intersect each other, then these hyperedges form a partition of granules. Furthermore, if $S_{i}, S_{j} \in S$ and $S_{i} \cap S_{j} \neq \varnothing$, i.e., $S_{i}$ and $S_{j}$ intersect each other, then these hyperedges form a covering of granules at this level. Note that, the definition of $q$-RPFH concludes that the $q$-RPFH forms a covering of a set of universes $X$.

Example 3. Let $X=\left\{x_{1}, x_{2}, x_{3}, x_{4}, x_{5}, x_{6}, x_{7}, x_{8}, x_{9}, x_{10}\right\}$. The partition and covering of $X$ is given in Figures 5 and 6 , respectively.

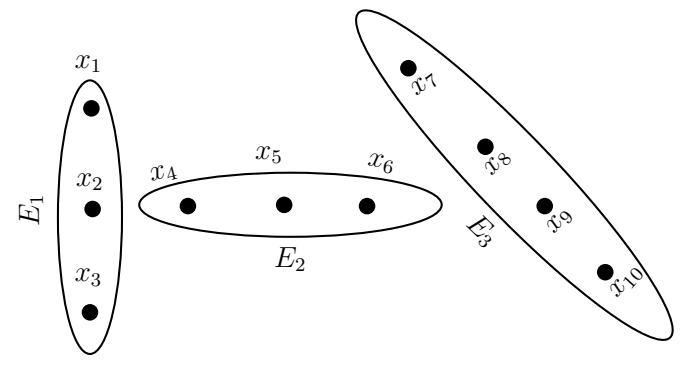

Figure 5. A partition of granules in a level.

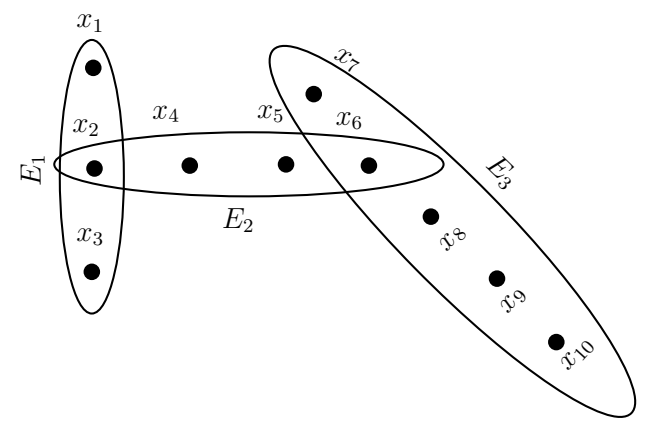

Figure 6. A covering of granules in a level.

The elements belonging to the granule determine the internal property and study the relationships between objects. The internal property in this model incorporates the interaction of vertices. The external property reflects the relationships between granules. This property incorporates those vertices that belong to a $q$-RPF hyperedge under the membership functions $T, N$, and $F$. The existence of a granule is indicated by the contextual property in an environment.

A set-theoretic way to study the GrC model uses the following operators in a $q$-RPFH model. 
Definition 22. Let $\mathcal{G}_{1}$ and $\mathcal{G}_{2}$ be two granules in our model and the $q$-RPF hyperedges $\mathcal{E}_{1}$, $\mathcal{E}_{2}$ represent their external properties. The union of two granules $\mathcal{G}_{1} \cup \mathcal{G}_{2}$ is defined as a larger $q$-RPF hyperedge that contains the vertices of both $\mathcal{E}_{1}$ and $\mathcal{E}_{2}$. If $x_{i} \in \mathcal{G}_{1} \cup \mathcal{G}_{2}$, then the membership degrees of $x_{i}$ in larger granule $\mathcal{G}_{1} \cup \mathcal{G}_{2}$ are defined as follows,

$$
\begin{aligned}
& T_{\mathcal{G}_{1} \cup \mathcal{G}_{2}}\left(x_{i}\right)= \begin{cases}\max \left\{T_{\mathcal{E}_{1}}\left(x_{i}\right), T_{\mathcal{E}_{2}}\left(x_{i}\right)\right\}, & \text { if } \quad x_{i} \in \mathcal{E}_{1} \text { and } x_{i} \in \mathcal{E}_{2} \\
T_{\mathcal{E}_{1}}\left(x_{i}\right), & \text { if } \quad x_{i} \in \mathcal{E}_{1} \text { and } x_{i} \notin \mathcal{E}_{2} \\
T_{\mathcal{E}_{2}}\left(x_{i}\right), & \text { if } \quad x_{i} \in \mathcal{E}_{2} \text { and } x_{i} \notin \mathcal{E}_{1}\end{cases} \\
& N_{\mathcal{G}_{1} \cup \mathcal{G}_{2}}\left(x_{i}\right)= \begin{cases}\max \left\{N_{\mathcal{E}_{1}}\left(x_{i}\right), N_{\mathcal{E}_{2}}\left(x_{i}\right)\right\}, & \text { if } \quad x_{i} \in \mathcal{E}_{1} \text { and } x_{i} \in \mathcal{E}_{2}, \\
N_{\mathcal{E}_{1}}\left(x_{i}\right), & \text { if } x_{i} \in \mathcal{E}_{1} \text { and } x_{i} \notin \mathcal{E}_{2}, \\
N_{\mathcal{E}_{2}}\left(x_{i}\right), & \text { if } \quad x_{i} \in \mathcal{E}_{2} \text { and } x_{i} \notin \mathcal{E}_{1} .\end{cases} \\
& F_{\mathcal{G}_{1} \cup \mathcal{G}_{2}}\left(x_{i}\right)= \begin{cases}\min \left\{F_{\mathcal{E}_{1}}\left(x_{i}\right), F_{\mathcal{E}_{2}}\left(x_{i}\right)\right\}, & \text { if } \quad x_{i} \in \mathcal{E}_{1} \text { and } x_{i} \in \mathcal{E}_{2}, \\
F_{\mathcal{E}_{1}}\left(x_{i}\right), & \text { if } x_{i} \in \mathcal{E}_{1} \text { and } x_{i} \notin \mathcal{E}_{2}, \\
F_{\mathcal{E}_{2}}\left(x_{i}\right), & \text { if } \quad x_{i} \in \mathcal{E}_{2} \text { and } x_{i} \notin \mathcal{E}_{1}\end{cases}
\end{aligned}
$$

Definition 23. Let $\mathcal{G}_{1}$ and $\mathcal{G}_{2}$ be two granules in our model and the $q$-RPF hyperedges $\mathcal{E}_{1}$, $\mathcal{E}_{2}$ represent their external properties. The intersection of two granules $\mathcal{G}_{1} \cap \mathcal{G}_{2}$ is defined as a smaller $q$-RPF hyperedge that contains those vertices belonging to both $\mathcal{E}_{1}$ and $\mathcal{E}_{2}$. If $x_{i} \in \mathcal{G}_{1} \cap \mathcal{G}_{2}$, then the membership degrees of $x_{i}$ in smaller granule $\mathcal{G}_{1} \cap \mathcal{G}_{2}$ are defined as follows,

$$
\begin{gathered}
T_{\mathcal{G}_{1} \cap \mathcal{G}_{2}}\left(x_{i}\right)= \begin{cases}\min \left\{T_{\mathcal{E}_{1}}\left(x_{i}\right), T_{\mathcal{E}_{2}}\left(x_{i}\right)\right\}, & \text { if } x_{i} \in \mathcal{E}_{1} \text { and } x_{i} \in \mathcal{E}_{2}, \\
T_{\mathcal{E}_{1}}\left(x_{i}\right), & \text { if } x_{i} \in \mathcal{E}_{1} \text { and } x_{i} \notin \mathcal{E}_{2}, \\
T_{\mathcal{E}_{2}}\left(x_{i}\right), & \text { if } \quad x_{i} \in \mathcal{E}_{2} \text { and } x_{i} \notin \mathcal{E}_{1} .\end{cases} \\
N_{\mathcal{G}_{1} \cap \mathcal{G}_{2}}\left(x_{i}\right)= \begin{cases}\min \left\{N_{\mathcal{E}_{1}}\left(x_{i}\right), N_{\mathcal{E}_{2}}\left(x_{i}\right)\right\}, & \text { if } \quad x_{i} \in \mathcal{E}_{1} \text { and } x_{i} \in \mathcal{E}_{2}, \\
N_{\mathcal{E}_{1}}\left(x_{i}\right), & \text { if } \quad x_{i} \in \mathcal{E}_{1} \text { and } x_{i} \notin \mathcal{E}_{2}, \\
N_{\mathcal{E}_{2}}\left(x_{i}\right), & \text { if } \quad x_{i} \in \mathcal{E}_{2} \text { and } x_{i} \notin \mathcal{E}_{1} .\end{cases} \\
F_{\mathcal{G}_{1} \cap \mathcal{G}_{2}}\left(x_{i}\right)= \begin{cases}\max \left\{F_{\mathcal{E}_{1}}\left(x_{i}\right), F_{\mathcal{E}_{2}}\left(x_{i}\right)\right\}, & \text { if } \quad x_{i} \in \mathcal{E}_{1} \text { and } x_{i} \in \mathcal{E}_{2}, \\
F_{\mathcal{E}_{1}}\left(x_{i}\right), & \text { if } \quad x_{i} \in \mathcal{E}_{1} \text { and } x_{i} \notin \mathcal{E}_{2}, \\
F_{\mathcal{E}_{2}}\left(x_{i}\right), & \text { if } \quad x_{i} \in \mathcal{E}_{2} \text { and } x_{i} \notin \mathcal{E}_{1} .\end{cases}
\end{gathered}
$$

Definition 24. Let $\mathcal{G}_{1}$ and $\mathcal{G}_{2}$ be two granules in our model and the $q$-RPF hyperedges $\mathcal{E}_{1}, \mathcal{E}_{2}$ represent their external properties. The difference between two granules $\mathcal{G}_{1}-\mathcal{G}_{2}$ is defined as a smaller $q$-RPF hyperedge that contains those vertices belonging to $\mathcal{E}_{1}$, but not to $\mathcal{E}_{2}$.

Note that, if a vertex $x_{i} \in \mathcal{E}_{1}$ and $x_{i} \notin \mathcal{E}_{2}$, then:

$$
T_{\mathcal{E}_{1}}\left(x_{i}\right)>0, N_{\mathcal{E}_{1}}\left(x_{i}\right)>0, F_{\mathcal{E}_{1}}\left(x_{i}\right)<1 \text { and } T_{\mathcal{E}_{2}}\left(x_{i}\right)=0, N_{\mathcal{E}_{2}}\left(x_{i}\right)=0, F_{\mathcal{E}_{2}}\left(x_{i}\right)=1
$$

Definition 25. A granule $\mathcal{G}_{1}$ is said to be the sub-granule of $\mathcal{G}_{2}$, if each vertex $x_{i}$ of $\mathcal{E}_{1}$ also belongs to $\mathcal{E}_{2}$, i.e., $\mathcal{E}_{1} \subseteq \mathcal{E}_{2}$. In such a case, $\mathcal{G}_{2}$ is called the super-granule of $\mathcal{G}_{1}$.

Note that, if $\mathcal{E}\left(x_{i}\right)=\{0,1\}$, then all above-described operators are reduced to the classical hypergraphs theory of GrC. 


\subsection{The Construction of Hierarchical Structures}

As earlier, we have constructed a granular structure of a specific level as a $q$-RPFH. In this way, we can interpret a problem at distinct levels of granularities. Hence, these granular structures at different levels produce a set of $q$-RPFHs. The upper set of these hypergraphs constructs a hierarchical structure at distinct levels. The relationships between granules are expressed by the lower level, which represents the problem as a concrete example of granularity. The relationships between granule sets are expressed by the higher level, which represents the problem as an abstract example of granularity. Thus, the single-level structures can be constructed and then can be subdivided into hierarchical structures using the relational mappings between different levels.

Definition 26. Let $H_{1}=\left(\mathcal{R}_{1}, S_{1}\right)$ and $H_{2}=\left(\mathcal{R}_{2}, S_{2}\right)$ be two q-RPFHs. In a hierarchy structure, their level cuts are $H_{1}^{(\alpha, \beta, \gamma)}$ and $H_{2}^{(\alpha, \beta, \gamma)}$, respectively. Let $(\alpha, \beta, \gamma) \in[0,1]$ and $T_{\mathcal{E}_{i}^{1}} \geq \alpha, N_{\mathcal{E}_{i}^{1}} \geq \beta, F_{\mathcal{E}_{i}^{1}} \leq \gamma$, where $\mathcal{E}_{i}^{1} \in S_{1}$, then a mapping $\phi: H_{1}^{(\alpha, \beta, \gamma)} \rightarrow H_{2}^{(\alpha, \beta, \gamma)}$ from $H_{1}^{(\alpha, \beta, \gamma)}$ to $H_{2}^{(\alpha, \beta, \gamma)}$ maps the $\mathcal{E}_{i}^{1(\alpha, \beta, \gamma)}$ in $H_{1}^{(\alpha, \beta, \gamma)}$ to a vertex $x_{i}^{2}$ in $H_{2}^{(\alpha, \beta, \gamma)}$. Furthermore, the mapping $\phi^{-1}: H_{2}^{(\alpha, \beta, \gamma)} \rightarrow H_{1}^{(\alpha, \beta, \gamma)}$ maps a vertex $x_{i}^{2}$ in $H_{2}^{(\alpha, \beta, \gamma)}$ to the $(\alpha, \beta, \gamma)$-cut of $q$-RPF hyperedge $\mathcal{E}_{i}^{1(\alpha, \beta, \gamma)}$ in $H_{1}^{(\alpha, \beta, \gamma)}$. It can be denoted as $\phi\left(\mathcal{E}_{i}^{1(\alpha, \beta, \gamma)}\right)=x_{i}^{2}$ or $\phi^{-1}\left(x_{i}^{2}\right)=\mathcal{E}_{i}^{1(\alpha, \beta, \gamma)}$, for $1 \leq i \leq n$.

The $q$-RPFH $H_{1}$ possesses a finer granularity than $H_{2}$, so $H_{1}$ is referred to as the finer granularity and $\mathrm{H}_{2}$ as the coarser granularity.

In a $q$-RPFH model, the mappings are used to describe the relations among different levels of granularities. At each distinct level, the problem is interpreted w.r.t. the $q$-RPF granularity of that level. The different descriptions of some problems are associated through mappings at distinct levels of granularities. There are two fundamental types to construct the hierarchical structures, the top-down construction procedure and the bottom-up construction procedure [34]. The method for the bottom-up construction is described in Algorithm 1.

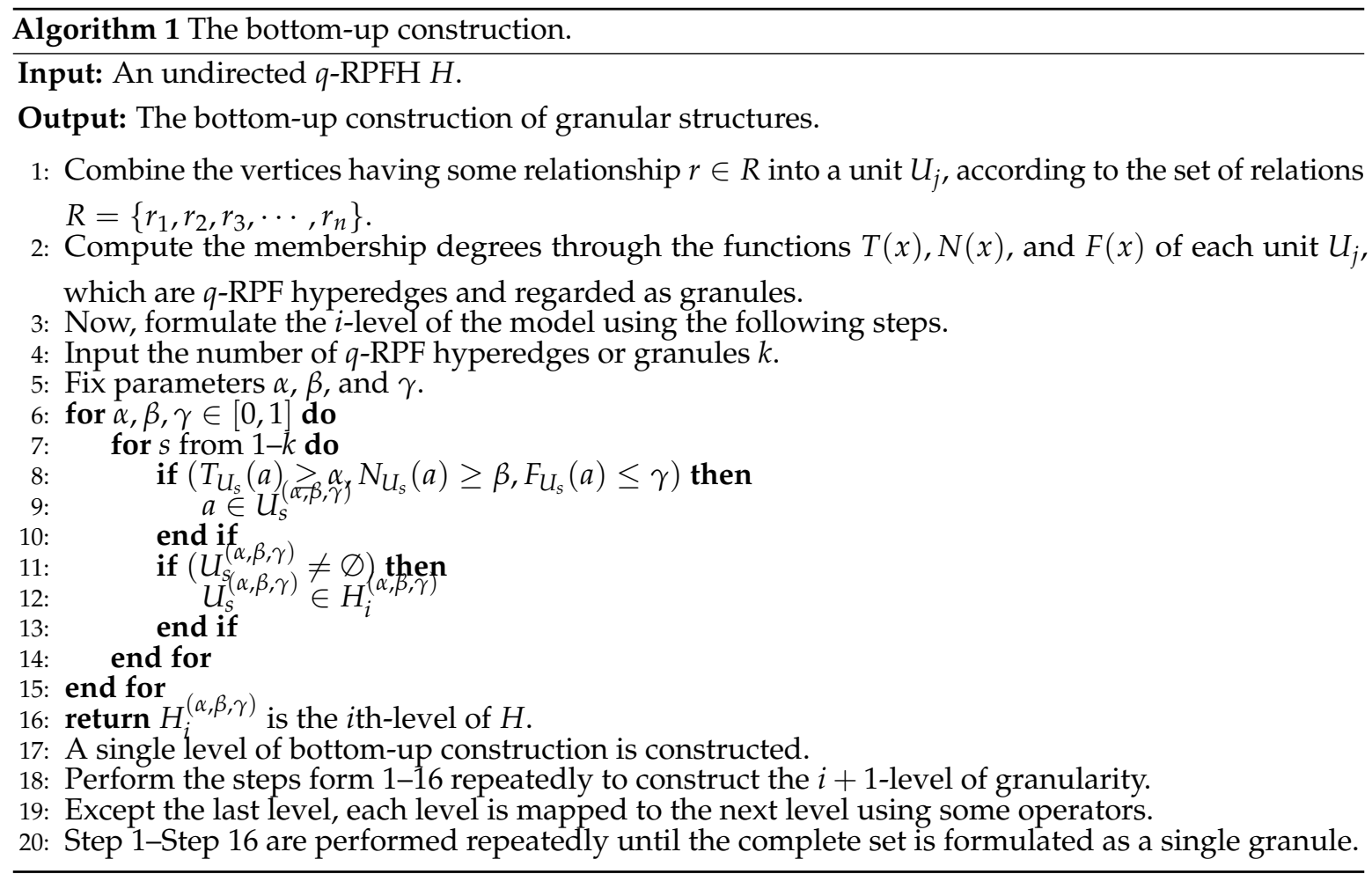

The method of bottom-up construction is explained by the following example. 
Example 4. Let $H=(\mathcal{R}, S)$ be a seven-rung picture fuzzy hypergraph as shown in Figure 7 . Let $X=$ $\left\{x_{1}, x_{2}, x_{3}, x_{4}, x_{5}, x_{6}, x_{7}, x_{8}, x_{9}, x_{10}, x_{11}, x_{12}\right\}$ and $S=\left\{\mathcal{E}_{1}, \mathcal{E}_{2}, \mathcal{E}_{3}, \mathcal{E}_{4}, \mathcal{E}_{5}\right\}$.

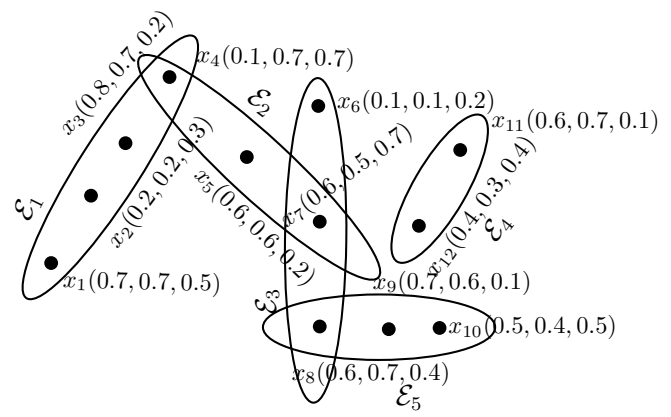

Figure 7. A seven-rung picture fuzzy hypergraph (RPFH).

For $\alpha=0.5, \beta=0.5$, and $\gamma=0.6$, the $(0.5,0.5,0.6)$-level hypergraph of $H$ is given in Figure 8 .

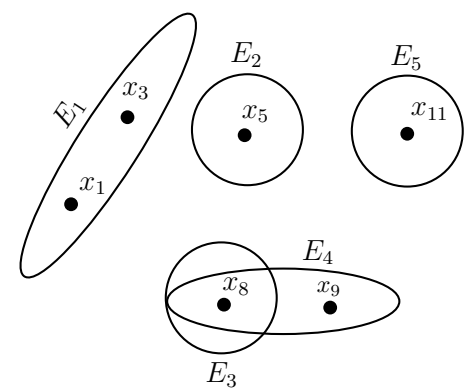

Figure 8. The $(0.5,0.5,0.6)$-level hypergraph of $H$.

By considering the fixed $\alpha, \beta, \gamma$ and following Algorithm 1, the bottom-up construction of this model is given in Figure 9.

In $\mathrm{GrC}$, more than one hierarchical structure (HS) is considered to emphasize the multiple approaches. These different HSs can be formed by considering the different interpretations of the relation set $R$. Every HS is a distinct aspect of the problem. A multiple or a multilevel model of $\mathrm{GrC}$ based on the hypergraph is formed by combining these different hierarchical structures. In a hypergraph model, a series of correlated hypergraphs is used to present every hierarchical structure. The mapping relates the distinct levels in an HS, and each hypergraph denotes a particular level in that structure. Each specific level has multiple hyperedges, and the objects having similar attributes are contained in one hyperedge. We now extend the example given in [23] to construct a $q$-RPFH model of GrC to illustrate the validity and flexibility of our model.

Example 5. Consider an express hypernetwork, where the vertices represent the express corporations. These vertices are combined together in one unit $U$ according to the relation set $R$ and possessing some type of relation among them. To form a 6-RPF hyperedge, which is also called a granule, we calculate and assign the membership degrees to each unit. Each 6-RPF hyperedge denotes a shop demanding express services, and the vertices contained in that hyperedge are the express corporations serving that shop. There are ten $\left\{c_{1}, c_{2}, c_{3}, c_{4}, c_{5}, c_{6}, c_{7}, c_{8}, c_{9}, c_{10}\right\}$ express corporations, and the corresponding incidence matrix of this model is given in Table 2. 


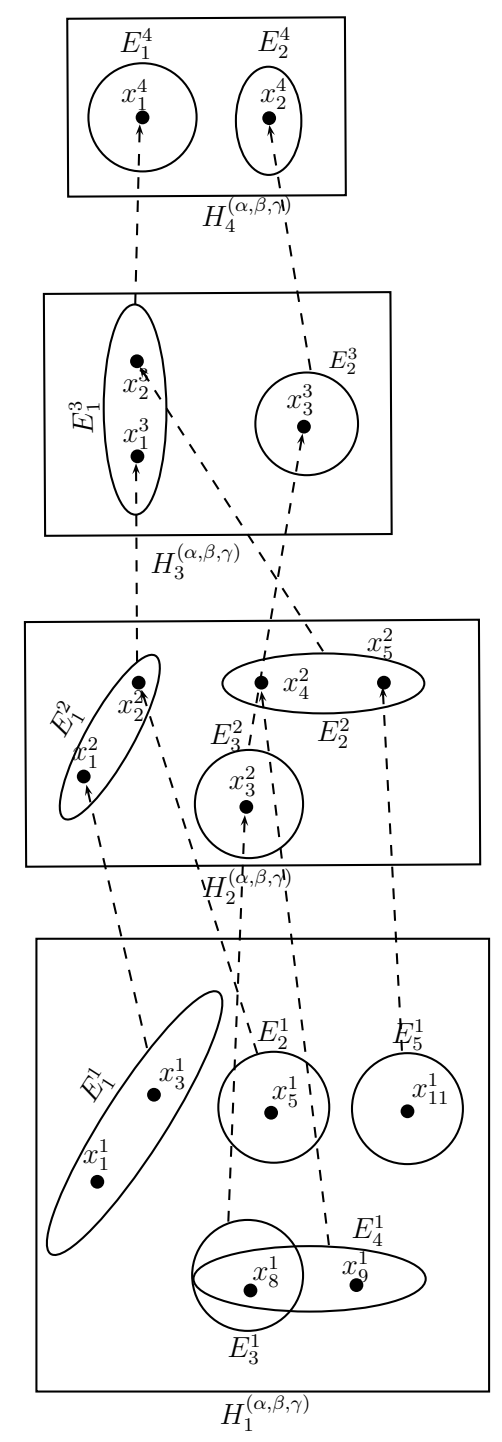

Figure 9. The bottom-up construction procedure.

Table 2. Incidence matrix of the express hypernetwork.

\begin{tabular}{llllll}
\hline & Shop 1 & Shop 2 & Shop 3 & Shop 4 & Shop 5 \\
\hline$c_{1}$ & $(0.8,0.2,0.1)$ & $(0,0,1)$ & $(0,0,1)$ & $(0,0,1)$ & $(0,0,1)$ \\
$c_{2}$ & $(0.9,0.3,0.5)$ & $(0,0,1)$ & $(1,0,0)$ & $(0,0,1)$ & $(0,0,1)$ \\
$c_{3}$ & $(0.3,0.5,0.9)$ & $(0.6,0.5,0.2)$ & $(0,0,1)$ & $(0,0,1)$ & $(0,0,1)$ \\
$c_{4}$ & $(0.8,0.2,0.1)$ & $(0,0,1)$ & $(0,0,1)$ & $(0,0,1)$ & $(0,0,1)$ \\
$c_{5}$ & $(0,0,1)$ & $(0.3,0.5,0.9)$ & $(0,0,1)$ & $(0,0,1)$ & $(0,0,1)$ \\
$c_{6}$ & $(0,0,1)$ & $(0.7,0.2,0.3)$ & $(0,0,1)$ & $(0,0,1)$ & $(0,0,1)$ \\
$c_{7}$ & $(0,0,1)$ & $(0.5,0.3,0.6)$ & $(0,0,1)$ & $(0.1,0.2,0.8)$ & $(0,0,1)$ \\
$c_{8}$ & $(0,0,1)$ & $(0,0,1)$ & $(0,0,1)$ & $(0.6,0.5,0.2)$ & $(0,0,1)$ \\
$c_{9}$ & $(0,0,1)$ & $(0,0,1)$ & $(0.6,0.5,0.2)$ & $(0.7,0.2,0.3)$ & $(0.4,0.7,0.5)$ \\
$c_{10}$ & $(0,0,1)$ & $(0,0,1)$ & $(0,0,1)$ & $(0,0,1)$ & $(0.5,0.2,0.7)$ \\
\hline
\end{tabular}

The corresponding 6-rung picture fuzzy hypergraph is shown in Figure 10. 


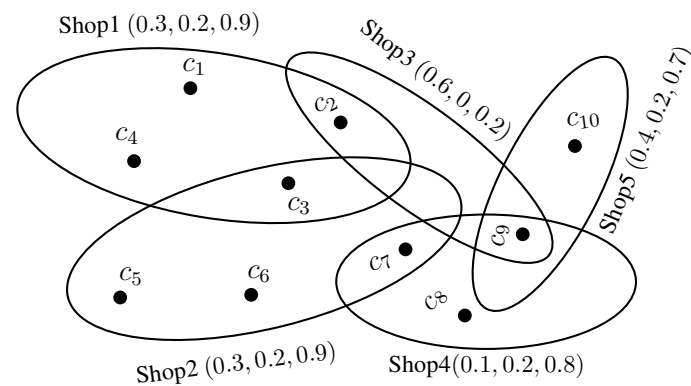

Figure 10. A 6-rung picture fuzzy hypergraph model of express corporations.

A 6-RPFH model of GrC illustrates an uncertain set of objects with specific positive, neutral, and negative degrees, which incorporate the undetermined behavior of an element with respect to its granule. In this example, there are five shops requiring the services of the express corporation. The membership degrees of each corporation to distinct shops is different because every shop selects a corporation by considering different factors, including consignment, life span, limited liability, scale of that express corporation, the distance of receiving and mailing parcels, and so on. Note that, using a q-RPFH in GrC is meaningful and more flexible as compared to the fuzzy hypergraph because it also deals with negative membership and the neutral behavior of objects toward their granules.

\subsection{Zooming-in and Zooming-out Operators}

The multi-level granularity of the problem is represented by a $q$-RPFH model, which allows the problem solvers to decompose it into various minor problems and transform it into other granularities. The transformation of the problem in other granularities is performed by using two operators, i.e, zooming-in and zooming-out operators. The transformation from the weaker level to the finer level of granularity is done by zoom-in and the zoom-out operators dealing with the shifting of the problem from coarser to finer granularity.

Definition 27. Let $H_{1}=\left(\mathcal{R}_{1}, S_{1}\right)$ and $H_{2}=\left(\mathcal{R}_{2}, S_{2}\right)$ be two q-RPFHs, which are considered as two levels of hierarchical structures, and $H_{2}$ owns a coarser granularity than $H_{1}$. Suppose $H_{1}^{(\alpha, \beta, \gamma)}=\left(X_{1}, E_{1}^{(\alpha, \beta, \gamma)}\right)$ and $H_{2}^{(\alpha, \beta, \gamma)}=\left(X_{2}, E_{2}^{(\alpha, \beta, \gamma)}\right)$ are the corresponding $(\alpha, \beta, \gamma)$-level hypergraphs of $H_{1}$ and $H_{2}$, respectively. Let $e_{i}^{1} \in E_{1}^{(\alpha, \beta, \gamma)}, x_{j}^{1} \in X_{1}, e_{j}^{2} \in E_{2}^{(\alpha, \beta, \gamma)}, x_{l}^{2}, x_{m}^{2} \in X_{2}$, and $x_{l}^{2}, x_{m}^{2} \in e_{j}^{2}$. If $\phi\left(e_{i}^{1}\right)=x_{l}^{2}$, then $r\left(x_{j}^{1}, x_{m}^{2}\right)$ is the relationship between $x_{j}^{1}$ and $x_{m}^{2}$ and is obtained by the characteristics of granules.

Definition 28. Let the hyperedge $\phi^{-} 1\left(x_{l}\right)$ be a vertex at a new level and the relation between hyperedges at this level be the same as that of the relationship between vertices at the previous level. This is called the zoom-in operator and transforms a weaker level to a stronger level. The function $r\left(x_{j}^{1}, x_{m}^{2}\right)$ defines the relation between vertices of the original level, as well as the new level.

Let the vertex $\phi\left(e_{i}\right)$ be a hyperedge at the next level and the relation between elements at this level be the same as that of the relationship between hyperedges at the corresponding level. This is called the zoom-out operator and transformation of a finer granularity to a coarser one.

By applying these operators, a problem can be viewed at multiple levels of granularities. These operations allow us to solve the problem more appropriately, and granularity can be switched easily at any level of problem solving.

In a $q$-RPFH model of GrC, the membership degrees of elements reflect the actual situation more efficiently, and a wide variety of complicated problems in uncertain and vague environments can be presented by means of $q$-RPFHs. The previous analysis concluded that this model of GrC generalized the classical hypergraph model and fuzzy hypergraph model.

We now construct a hypergraph model of GrC based on $q$-RPFER. 


\section{A Level Hypergraph Partition Model}

In this partition model, the blocks are represented by hyperedges and each block contains the elements having some $q$-RPFER $R$. Let $\pi_{R}$ be the partition of $X$ induced by $R$, then the $q$-RPFER $R_{\pi}$ is defined as, $x R_{\pi} y$ if and only if they are contained in a similar block/subset.

Definition 29. A system $\left(X, R^{\rho}\right), \rho=(\alpha, \beta, \gamma)$ is called an object space, where $R^{\rho}$ is a non-empty collection of equivalence relations between the elements of $X . R^{\rho}=\left\{r_{1}^{\rho}, r_{2}^{\rho}, r_{3}^{\rho}, \cdots, r_{n}^{\rho}\right\}, n=|X|$. For $i \leq n, r_{i}^{\rho} \in R^{\rho}, r_{i}^{\rho} \subseteq$ $X \times X \times X \cdots \times X$, if $\left(x_{1}, x_{2}, \cdots, x_{i}\right) \subseteq r_{i}^{\rho}$, then there exists an i-array relation $r_{i}^{\rho}$ on $\left(x_{1}, x_{2}, \cdots, x_{i}\right)$.

Example 6. Let $X=\left\{x_{1}, x_{2}, x_{3}, x_{4}, x_{5}\right\}$ and $R^{\rho}=\left\{r_{1}^{\rho}, r_{2}^{\rho}, r_{3}^{\rho}, r_{4}^{\rho}, r_{5}^{\rho}\right\}$, for $0.7<\alpha \leq 0.9,0.5<\beta \leq$ $0.5,0.1>\gamma \geq 0.1$, as shown in Example 1, $r_{1}^{\rho}=\left\{\left(x_{1}\right),\left(x_{2}\right),\left(x_{3}\right)\right\}, r_{2}^{\rho}=\left\{\left(x_{4}, x_{5}\right)\right\}, r_{3}^{\rho}, r_{4}^{\rho}, r_{5}^{\rho}=\{\varnothing\}$.

The $q$-RPFERs between the vertices are obtained from the actual situation, and then, the vertices having some relation are combined into a hyperedge. After the integration of all vertices into hyperedges is done, a single level of the GrC model is obtained. The construction of the level hypergraph model is illustrated through the following example, which is the extension of example given in [23].

Example 7. Consider an express hypernetwork, as discussed in Example 5; we consider the same hypernetwork to illustrate the construction of the level hypergraph model. Ten express corporations are considered, and suppose that each corporation experiences six attributes. These attributes are delivery time, freight, tracking and circulation information, time window service, customer satisfaction, and flexible and customizable and are denoted by $A_{1}, A_{2}, A_{3}, A_{4}, A_{5}$, and $A_{6}$, respectively. To indicate the values of attributes, zero and one are used, as shown in Table 3.

Table 3. Description of the attributes.

\begin{tabular}{lllll}
\hline Attributes & Characteristic & Value & Characteristic & Value \\
\hline Delivery time & Short & 1 & Long & 0 \\
Freight & Low & 1 & High & 0 \\
Tracking and circulation information & Existence & 1 & Non-existence & 0 \\
Time window service & Existence & 1 & Non-existence & 0 \\
Customer satisfaction & High & 1 & Low & 0 \\
Flexible and customizable & Large & 1 & Small & 0 \\
\hline
\end{tabular}

The information about express corporations having these attributes is given in Table 4.

Table 4. Corporations having attributes.

\begin{tabular}{cccccccc}
\hline Express & Corporations & $\boldsymbol{A}_{\mathbf{1}}$ & $\boldsymbol{A}_{\mathbf{2}}$ & $\boldsymbol{A}_{\mathbf{3}}$ & $\boldsymbol{A}_{\mathbf{4}}$ & $\boldsymbol{A}_{\mathbf{5}}$ & $\boldsymbol{A}_{\mathbf{6}}$ \\
\hline$x_{1}$ & $E_{1}$ & 1 & 0 & 0 & 1 & 0 & 0 \\
$x_{2}$ & $E_{2}$ & 1 & 0 & 0 & 1 & 0 & 0 \\
$x_{3}$ & $E_{3}$ & 1 & 1 & 0 & 1 & 0 & 0 \\
$x_{4}$ & $E_{4}$ & 1 & 1 & 0 & 1 & 1 & 0 \\
$x_{5}$ & $E_{5}$ & 1 & 1 & 0 & 0 & 0 & 1 \\
$x_{6}$ & $E_{6}$ & 1 & 1 & 0 & 0 & 0 & 0 \\
$x_{7}$ & $E_{7}$ & 1 & 1 & 1 & 0 & 0 & 0 \\
$x_{8}$ & $E_{8}$ & 1 & 0 & 1 & 0 & 0 & 0 \\
$x_{9}$ & $E_{9}$ & 1 & 0 & 1 & 0 & 1 & 0 \\
$x_{10}$ & $E_{10}$ & 0 & 0 & 1 & 0 & 1 & 0 \\
\hline
\end{tabular}


The positive membership function $T\left(x_{i}\right) \in[0,1]$ represents the beneficial and helpful relationships between these corporations, and this affirmative relationship is described through the following matrix.

$$
T_{\tilde{M}_{R}}=\left[\begin{array}{cccccccccc}
1 & 1 & 0.67 & 0.55 & 0.25 & 0.33 & 0.25 & 0.33 & 0.25 & 0 \\
1 & 1 & 0.67 & 0.55 & 0.25 & 0.33 & 0.25 & 0.33 & 0.25 & 0 \\
0.67 & 0.67 & 1 & 0.75 & 0.55 & 0.66 & 0.55 & 0.25 & 0.20 & 0 \\
0.55 & 0.55 & 0.75 & 1 & 0.40 & 0.40 & 0.40 & 0.20 & 0.40 & 0.20 \\
0.25 & 0.25 & 0.55 & 0.40 & 1 & 0.67 & 0.55 & 0.25 & 0.20 & 0 \\
0.33 & 0.33 & 0.67 & 0.40 & 0.67 & 1 & 0.67 & 0.33 & 0.25 & 0 \\
0.25 & 0.25 & 0.55 & 0.40 & 0.55 & 0.67 & 1 & 0.67 & 0.55 & 0.25 \\
0.33 & 0.33 & 0.25 & 0.20 & 0.25 & 0.33 & 0.67 & 1 & 0.67 & 0.33 \\
0.25 & 0.25 & 0.20 & 0.40 & 0.20 & 0.25 & 0.55 & 0.67 & 1 & 0.67 \\
0 & 0 & 0 & 0.20 & 0 & 0 & 0.25 & 0.33 & 0.67 & 1
\end{array}\right] .
$$

The neutral membership function $N\left(x_{i}\right) \in[0,1]$ represents the unbiased and vague relationships between these corporations, and this indeterminate behavior is described through the following matrix.

$$
N_{\tilde{M}_{R}}=\left[\begin{array}{cccccccccc}
0 & 0 & 0.23 & 0.31 & 0.31 & 0.12 & 0.31 & 0.12 & 0.31 & 0 \\
0 & 0 & 0.23 & 0.31 & 0.31 & 0.12 & 0.31 & 0.12 & 0.31 & 0 \\
0.23 & 0.23 & 0 & 0.23 & 0.31 & 0.33 & 0.31 & 0.31 & 0.12 & 0 \\
0.31 & 0.31 & 0.23 & 0 & 0.20 & 0.20 & 0.20 & 0.12 & 0.20 & 0.12 \\
0.31 & 0.31 & 0.31 & 0.20 & 0 & 0.23 & 0.31 & 0.31 & 0.12 & 0 \\
0.12 & 0.12 & 0.23 & 0.20 & 0.23 & 0 & 0.23 & 0.12 & 0.31 & 0 \\
0.31 & 0.31 & 0.31 & 0.20 & 0.31 & 0.23 & 0 & 0.23 & 0.31 & 0.31 \\
0.12 & 0.12 & 0.31 & 0.12 & 0.31 & 0.12 & 0.23 & 0 & 0.23 & 0.12 \\
0.31 & 0.31 & 0.12 & 0.20 & 0.12 & 0.31 & 0.31 & 0.23 & 0 & 0.23 \\
0 & 0 & 0 & 0.12 & 0 & 0 & 0.31 & 0.12 & 0.23 & 0
\end{array}\right] .
$$

The negative membership function $F\left(x_{i}\right) \in[0,1]$ represents the contrary and competent relationships between these corporations, and this opposing relationship is described through the following matrix.

$$
F_{\tilde{M}_{R}}=\left[\begin{array}{cccccccccc}
0 & 0 & 0.32 & 0.45 & 0.65 & 0.65 & 0.65 & 0.65 & 0.65 & 1 \\
0 & 0 & 0.32 & 0.45 & 0.65 & 0.65 & 0.65 & 0.65 & 0.65 & 1 \\
0.32 & 0.32 & 0 & 0.25 & 0.45 & 0.23 & 0.45 & 0.65 & 0.7 & 1 \\
0.45 & 0.45 & 0.25 & 0 & 0.50 & 0.50 & 0.50 & 0.76 & 0.50 & 0.76 \\
0.65 & 0.65 & 0.45 & 0.50 & 0 & 0.32 & 0.45 & 0.65 & 0.76 & 1 \\
0.65 & 0.65 & 0.32 & 0.50 & 0.32 & 0 & 0.32 & 0.65 & 0.65 & 1 \\
0.65 & 0.65 & 0.45 & 0.50 & 0.45 & 0.32 & 0 & 0.32 & 0.45 & 0.65 \\
0.65 & 0.65 & 0.65 & 0.76 & 0.65 & 0.65 & 0.32 & 0 & 0.32 & 0.65 \\
0.65 & 0.65 & 0.76 & 0.50 & 0.76 & 0.65 & 0.45 & 0.32 & 0 & 0.32 \\
1 & 1 & 1 & 0.76 & 1 & 1 & 0.65 & 0.65 & 0.32 & 1
\end{array}\right] .
$$

A 5-RPFR matrix can be obtained by combining positive membership, neutral membership, and negative membership degrees, as shown in the following matrix.

$$
\tilde{M}_{R}=\left(\begin{array}{cccc}
(1,0,0) & (1,0,0) & \ldots & (0,0,1) \\
(1,0,0) & (1,0,0) & \ldots & (0,0,1) \\
(0.67,0.23,0.32) & (0.67,0.23,0.32) & \ldots & (0,0,1) \\
\vdots & \vdots & \vdots & \vdots \\
(0,0,1) & (0,0,1) & \ldots & (1,0,0)
\end{array}\right) .
$$


The positive $T\left(m_{i j}\right)$, neutral $N\left(m_{i j}\right)$, and negative $F\left(m_{i j}\right)$ degrees of each $m_{i j}$ entry of the above matrix describe the healthy, neutral, and rival relationships among the $x_{i}$ and $x_{j}$ corporations, respectively. The transitive closure of the above matrix is shown in the matrix below. The transitive closure of 5-RPFER is also an equivalence relation.
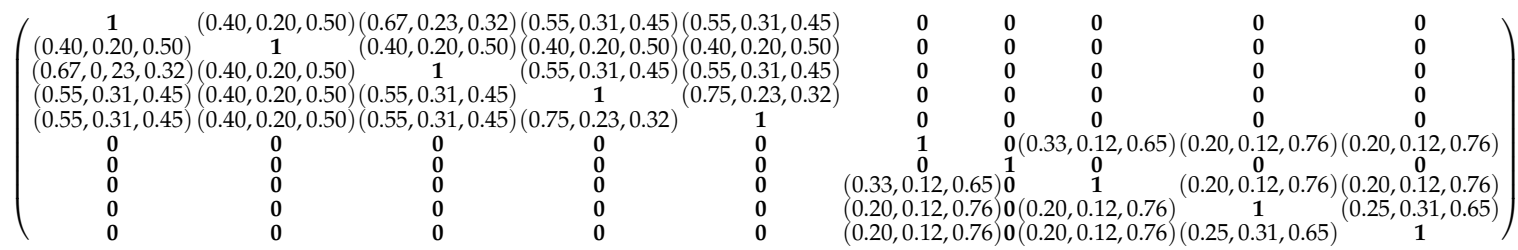

Note that, here, $\mathbf{0}=(0,0,1)$ and $\mathbf{1}=(1,0,0)$. The positive, neutral, and negative value domains are given as follows,

$T_{D}=\{0.75,0.67,0.55,0.40,0.33,0.25,0.20\}$,

$N_{D}=\{0.31,0.23,0.20,0.12\}$,

$F_{D}=\{0.76,0.65,0.50,0.45,0.32\}$.

Let $0.20<\alpha \leq 0.25,0.12<\beta \leq 0.20$, and $0.32<\gamma \leq 0.45$ and their corresponding HQS be given as follows,

$X / R^{(\alpha, \beta, \gamma)}=\left\{\left(x_{4}, x_{5}\right),\left(x_{1}, x_{4}, x_{5}\right),\left(x_{1}, x_{3}, x_{5}\right),\left(x_{1}, x_{3}, x_{4}\right)\right\}$.

Thus, we can conclude that $r_{1}=\{\varnothing\}, r_{2}=\left\{\left(x_{4}, x_{5}\right)\right\}, r_{3}=\left\{\left(x_{1}, x_{4}, x_{5}\right),\left(x_{1}, x_{3}, x_{5}\right),\left(x_{1}, x_{3}, x_{4}\right)\right\}, r_{4}$ $=r_{5}=r_{6}=r_{7}=r_{8}=r_{9}=r_{10}=\{\varnothing\}$. We obtain four hyperedges $E_{1}=\left\{x_{4}, x_{5}\right\}, E_{2}=\left\{x_{1}, x_{4}, x_{5}\right\}, E_{3}=$ $\left\{x_{1}, x_{3}, x_{5}\right\}, E_{4}=\left\{x_{1}, x_{3}, x_{4}\right\}$. Thus, we have constructed a single level of the hypergraph model, as shown in Figure 11.

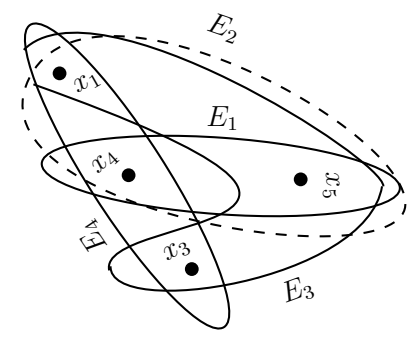

Figure 11. A single level of the hypergraph model.

It is noted that a $q$-RPFER determines the partition of the domain set into different layers. All $q$-RPFER, which are isomorphic, can also determine the same classification.

Definition 30. Let $H_{1}$ and $H_{2}$ be two crisp hypergraphs. Suppose that $H_{1}$ owns a finer q-RPF granularity than $\mathrm{H}_{2}$. A mapping from $\mathrm{H}_{1}$ to $\mathrm{H}_{2} \psi: \mathrm{H}_{1} \rightarrow \mathrm{H}_{2}$ maps a hyperedge of $\mathrm{H}_{1}$ to the vertex of $\mathrm{H}_{2}$, and the mapping $\psi^{-1}: H_{2} \rightarrow H_{1}$ maps a vertex of $H_{2}$ to the hyperedge of $H_{1}$.

The procedure of bottom-up construction for the level hypergraph model is illustrated in Algorithm 2. 


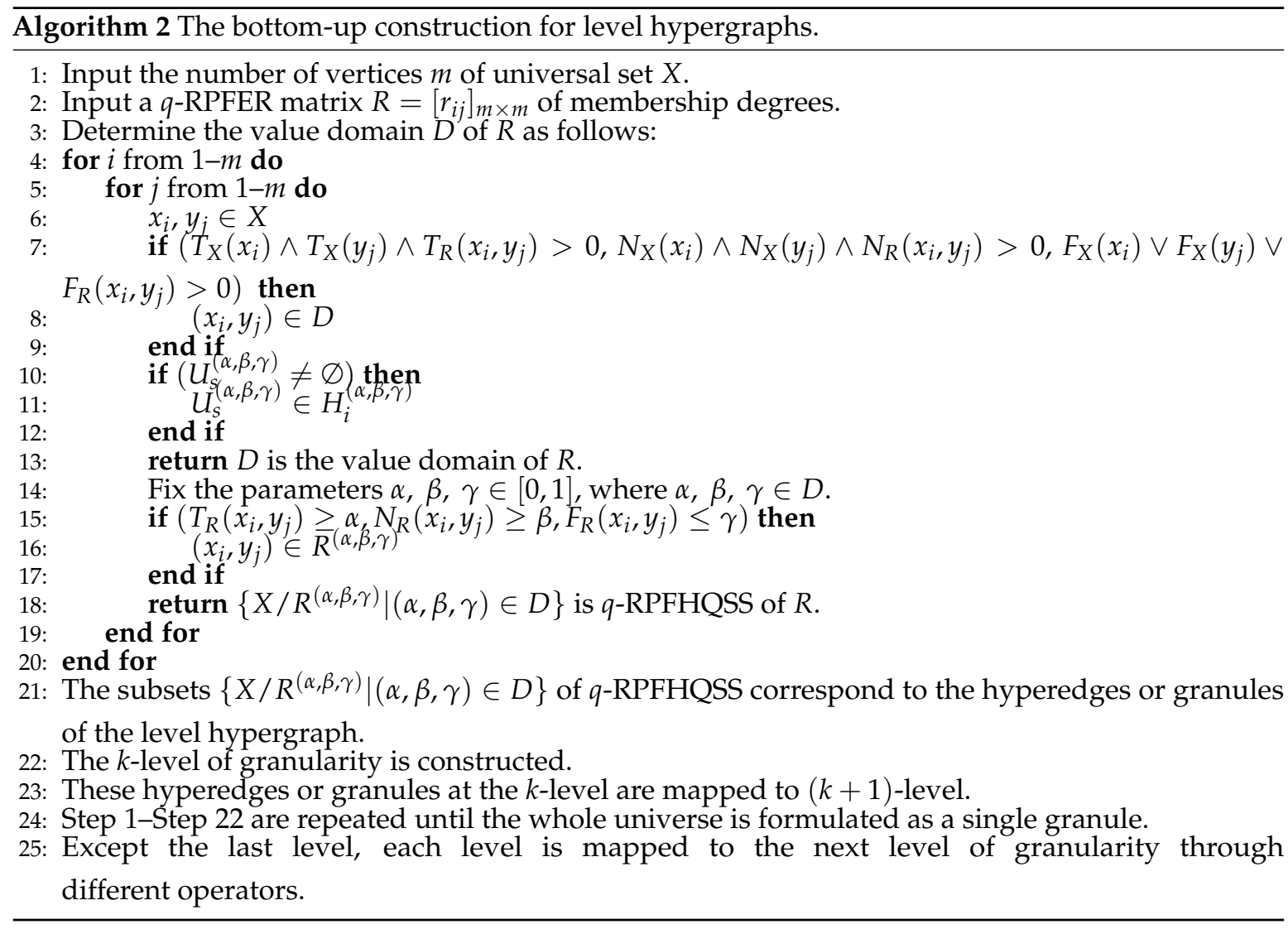

Definition 31. Let $R$ be a $q$-RPFER on $X$. A coarse grained universe $X / R^{(\alpha, \beta, \gamma)}$ can be obtained by using $q$-RPFER, where $\left[x_{i}\right]_{R^{\alpha, \beta, \gamma}}=\left\{x_{j} \in X \mid x_{i} R x_{j}\right\}$. This equivalence class $\left[x_{i}\right]_{R^{(\alpha, \beta, \gamma)}}$ is considered as a hyperedge in the level hypergraph.

Definition 32. Let $H_{1}=\left(X_{1}, E_{1}\right)$ and $H_{2}=\left(X_{2}, E_{2}\right)$ be level hypergraphs of $q-R P F H s$, and $H_{2}$ has weaker granularity than $H_{1}$. Suppose that $e_{i}^{1}, e_{j}^{2} \in E_{1}$, and $x_{i}^{2}, x_{j}^{2} \in X_{2}, i, j=1,2, \cdots, n$. The zoom-in operator $\kappa: H_{2} \rightarrow H_{1}$ is defined as $\kappa\left(x_{i}^{2}\right)=e_{i}^{1}, e_{i}^{1} \in E_{1}$. The relations between the vertices of $H_{2}$ define the relationships among the hyperedges at the new level. The zoom-in operator of the two levels is shown in Figure 12.

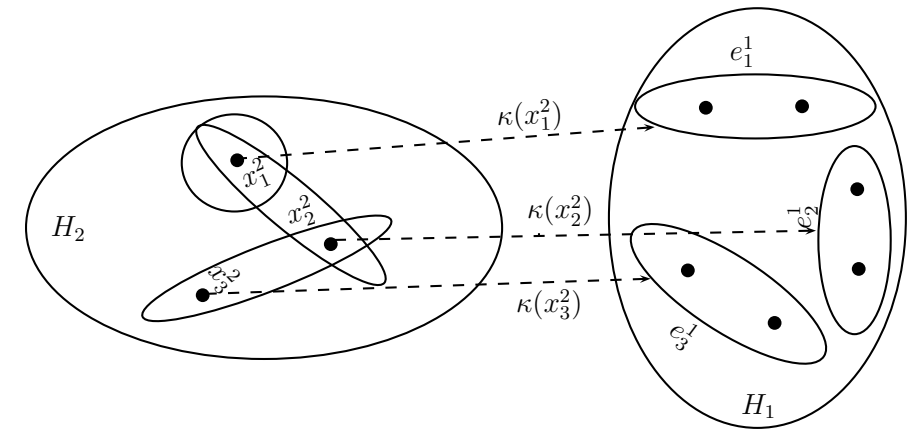

Figure 12. The zoom-in operator.

Remark 1. For all $X_{2}^{\prime}, X_{2}^{\prime \prime} \subseteq X_{2}$, we have $\kappa\left(X_{2}^{\prime}\right)=\bigcup_{x_{i}^{2} \in X_{2}^{\prime}} \kappa\left(x_{i}^{2}\right)$ and $\kappa\left(X_{2}^{\prime \prime}\right)=\underset{x_{j}^{2} \in X_{2}^{\prime \prime}}{\bigcup} \kappa\left(x_{j}^{2}\right)$.

Theorem 3. Let $H_{1}=\left(X_{1}, E_{1}\right)$ and $H_{2}=\left(X_{2}, E_{2}\right)$ be two levels and $\kappa: H_{2} \rightarrow H_{1}$ be the zoom-in operator. Then, for all $X_{2}^{\prime}, X_{2}^{\prime \prime} \subseteq X_{2}$, the zoom-in operator satisfies,

(i) $\kappa$ maps the empty set to an empty set, i.e., $\kappa(\varnothing)=\varnothing$. 
(ii) $\kappa\left(X_{2}\right)=E_{1}$.

(iii) $\kappa\left(\left[X_{2}^{\prime}\right]^{c}\right)=\left[\kappa\left(X_{2}^{\prime}\right)\right]^{c}$.

(iv) $\kappa\left(X_{2}^{\prime} \cap X_{2}^{\prime \prime}\right)=\kappa\left(X_{2}^{\prime}\right) \cap \kappa\left(X_{2}^{\prime \prime}\right)$.

(v) $\kappa\left(X_{2}^{\prime} \cup X_{2}^{\prime \prime}\right)=\kappa\left(X_{2}^{\prime}\right) \cup \kappa\left(X_{2}^{\prime \prime}\right)$.

(vi) $X_{2}^{\prime} \subseteq X_{2}^{\prime \prime}$ if and only if $\kappa\left(X_{2}^{\prime}\right) \subseteq \kappa\left(X_{2}^{\prime \prime}\right)$.

Proof. (i) It is trivially satisfied that $\kappa(\varnothing)=\varnothing$.

(ii) As we know that for all $x_{i}^{2} \in X_{2}$, we have $\kappa\left(X_{2}^{\prime}\right)=\underset{x_{i}^{2} \in X_{2}^{\prime}}{\bigcup} \kappa\left(x_{i}^{2}\right)$. Since $\kappa\left(x_{i}^{2}\right)=e_{i}^{1}$; thus, we have $\kappa\left(X_{2}^{\prime}\right)=\bigcup_{x_{i}^{2} \in X_{2}^{\prime}} \kappa\left(x_{i}^{2}\right)=\bigcup_{e_{i}^{1} \in E_{1}} e_{i}^{1}=E_{1}$.

(iii) Let $\left[X_{2}^{\prime}\right]^{c}=Z_{2}^{\prime}$ and $\left[X_{2}^{\prime \prime}\right]^{c}=Z_{2}^{\prime \prime}$, then it is obvious that $Z_{2}^{\prime} \cap X_{2}^{\prime}=\varnothing$ and $Z_{2}^{\prime} \cup X_{2}^{\prime}=X_{2}$. It follows from (ii) that $\kappa\left(X_{2}\right)=E_{1}$, and we denote by $W_{1}^{\prime}$ that edge set of $H_{1}$ on which the vertex set $Z_{2}^{\prime}$ of $H_{2}$ is mapped under $\kappa$, i.e., $\kappa\left(Z_{2}^{\prime}\right)=W_{1}^{\prime}$. Then, $\kappa\left(\left[X_{2}^{\prime}\right]^{c}\right)=\kappa\left(Z_{2}^{\prime}\right)=\bigcup_{x_{i}^{2} \in Z_{2}^{\prime}} \kappa\left(x_{i}^{2}\right)=\bigcup_{e_{i}^{1} \in W_{1}^{\prime}} e_{i}^{1}=Z_{1}^{\prime}$ and $\left[\kappa\left(X_{2}^{\prime}\right)\right]^{c}=\left[\bigcup_{x_{j}^{2} \in X_{2}^{\prime}} \kappa\left(x_{j}^{2}\right)\right]^{c}=\left[\bigcup_{e_{j}^{1} \in E_{1}^{\prime}} e_{j}^{1}\right]^{c}=\left(E_{1}^{\prime}\right)^{c}$. Since the relationship between hyperedges at the new level is the same as that of the relations among the vertices at the original level, we have $\left(E_{1}^{\prime}\right)^{c}=Z_{1}^{\prime}$. Hence, we conclude that $\kappa\left(\left[X_{2}^{\prime}\right]^{c}\right)=\left[\kappa\left(X_{2}^{\prime}\right)\right]^{c}$.

(iv) Assume that $X_{2}^{\prime} \cap X_{2}^{\prime \prime}=\tilde{X}_{2}$, then for all $x_{i}^{2} \in \tilde{X}_{2}$, this implies that $x_{i}^{2} \in X_{2}^{\prime}$ and $x_{i}^{2} \in X_{2}^{\prime \prime}$. Further, we have $\kappa\left(X_{2}^{\prime} \cap X_{2}^{\prime \prime}\right)=\kappa\left(\tilde{X}_{2}\right)=\bigcup_{x_{i}^{2} \in \tilde{X}_{2}} \kappa\left(x_{i}^{2}\right)=\bigcup_{e_{i}^{1} \in \tilde{E_{1}}} \kappa\left(e_{i}^{1}\right)=\tilde{E_{1}} \cdot \kappa\left(X_{2}^{\prime}\right) \cap \kappa\left(X_{2}^{\prime \prime}\right)=\left\{\bigcup_{x_{i}^{2} \in X_{2}^{\prime}} \kappa\left(x_{i}^{2}\right)\right\}$ $\cap\left\{\bigcup_{x_{j}^{2} \in X_{2}^{\prime \prime}} \kappa\left(x_{j}^{2}\right)\right\}=\bigcup_{e_{i}^{1} \in E_{1}^{\prime}} e_{i}^{1} \cap \bigcup_{e_{j}^{1} \in E_{1}^{\prime \prime}} e_{j}^{1}=E_{1}^{\prime} \cap E_{1}^{\prime \prime}$. Since the relationship between hyperedges at the new level is the same as that of the relations among the vertices at the original level, we have $E_{1}^{\prime} \cap E_{1}^{\prime \prime}=\tilde{E_{1}}$. Hence, we conclude that $\kappa\left(X_{2}^{\prime} \cap X_{2}^{\prime \prime}\right)=\kappa\left(X_{2}^{\prime}\right) \cap \kappa\left(X_{2}^{\prime \prime}\right)$.

(v) Assume that $X_{2}^{\prime} \cup X_{2}^{\prime \prime}=\bar{X}_{2}$. Then, we have $\kappa\left(X_{2}^{\prime} \cup X_{2}^{\prime \prime}\right)=\kappa\left(\bar{X}_{2}\right)=\bigcup_{x_{i}^{2} \in \bar{X}_{2}} \kappa\left(x_{i}^{2}\right)=\bigcup_{e_{i}^{1} \in \bar{E}_{1}} \kappa\left(e_{i}^{1}\right)=\bar{E}_{1}$. $\kappa\left(X_{2}^{\prime}\right) \cup \kappa\left(X_{2}^{\prime \prime}\right)=\left\{\bigcup_{x_{i}^{2} \in X_{2}^{\prime}} \kappa\left(x_{i}^{2}\right)\right\} \cup\left\{\bigcup_{x_{j}^{2} \in X_{2}^{\prime \prime}} \kappa\left(x_{j}^{2}\right)\right\}=\bigcup_{e_{i}^{1} \in E_{1}^{\prime}} e_{i}^{1} \cup \bigcup_{e_{j}^{1} \in E_{1}^{\prime \prime}} e_{j}^{1}=E_{1}^{\prime} \cup E_{1}^{\prime \prime}$. Since the relationship between hyperedges at the new level is the same as that of the relations among the vertices at the original level, we have $E_{1}^{\prime} \cup E_{1}^{\prime \prime}=\bar{E}_{1}$. Hence, we conclude that $\kappa\left(X_{2}^{\prime} \cup X_{2}^{\prime \prime}\right)=$ $\kappa\left(X_{2}^{\prime}\right) \cup \kappa\left(X_{2}^{\prime \prime}\right)$.

(vi) First, we show that $X_{2}^{\prime} \subseteq X_{2}^{\prime \prime}$ implies that $\kappa\left(X_{2}^{\prime}\right) \subseteq \kappa\left(X_{2}^{\prime \prime}\right)$. Since $X_{2}^{\prime} \subseteq X_{2}^{\prime \prime}$, this implies that $X_{2}^{\prime} \cap X_{2}^{\prime \prime}=X_{2}^{\prime}$ and $\kappa\left(X_{2}^{\prime}\right)=\bigcup_{x_{i}^{2} \in X_{2}^{\prime}} \kappa\left(x_{i}^{2}\right)=\bigcup_{e_{i}^{1} \in E_{1}^{\prime}} e_{i}^{1}=E_{1}^{\prime}$. Furthermore, $\kappa\left(X_{2}^{\prime \prime}\right)=\bigcup_{x_{j}^{2} \in X_{2}^{\prime \prime}} \kappa\left(x_{j}^{2}\right)=$ $\bigcup_{e_{j}^{1} \in E_{1}^{\prime \prime}} e_{j}^{1}=E_{1}^{\prime \prime}$. Since the relationship between hyperedges at the new level is the same as that of the relations among the vertices at the original level, we have $E_{1}^{\prime} \subseteq E_{1}^{\prime \prime}$, i.e., $\kappa\left(X_{2}^{\prime}\right) \subseteq \kappa\left(X_{2}^{\prime \prime}\right)$. Hence, $X_{2}^{\prime} \subseteq X_{2}^{\prime \prime}$ implies that $\kappa\left(X_{2}^{\prime}\right) \subseteq \kappa\left(X_{2}^{\prime \prime}\right)$.

We now prove that $\kappa\left(X_{2}^{\prime}\right) \subseteq \kappa\left(X_{2}^{\prime \prime}\right)$ implies that $X_{2}^{\prime} \subseteq X_{2}^{\prime \prime}$. Suppose on the contrary that whenever $\kappa\left(X_{2}^{\prime}\right) \subseteq \kappa\left(X_{2}^{\prime \prime}\right)$, then there is at least one vertex $x_{i}^{2} \in X_{2}^{\prime}$, but $x_{i}^{2} \notin X_{2}^{\prime \prime}$, i.e., $X_{2}^{\prime} \nsubseteq X_{2}^{\prime \prime}$. Since, $\kappa\left(x_{i}^{2}\right)=e_{i}^{1}$, and the relationship between hyperedges at the new level is the same as that of the relations among the vertices at the original level, we have $e_{i}^{1} \in E_{1}^{\prime}$, but $e_{i}^{1} \notin E_{1}^{\prime \prime}$,.i.e., $E_{1}^{\prime} \nsubseteq E_{1}^{\prime \prime}$, which is a contradiction to the supposition. Thus, we have that $\kappa\left(X_{2}^{\prime}\right) \subseteq \kappa\left(X_{2}^{\prime \prime}\right)$ implies that $X_{2}^{\prime} \subseteq X_{2}^{\prime \prime}$. Hence, $X_{2}^{\prime} \subseteq X_{2}^{\prime \prime}$ if and only if $\kappa\left(X_{2}^{\prime}\right) \subseteq \kappa\left(X_{2}^{\prime \prime}\right)$.

Definition 33. Let $H_{1}=\left(X_{1}, E_{1}\right)$ and $H_{2}=\left(X_{2}, E_{2}\right)$ be level hypergraphs of $q$-RPFHs, and $H_{2}$ has weaker granularity than $H_{1}$. Suppose that $e_{i}^{1}, e_{j}^{2} \in E_{1}$, and $x_{i}^{2}, x_{j}^{2} \in X_{2}, i, j=1,2, \cdots, n$. The zoom-out operator $\sigma: H_{1} \rightarrow H_{2}$ is defined as $\sigma\left(e_{i}^{1}\right)=x_{i}^{2}, x_{i}^{2} \in X_{2}$. The zoom-out operator of two levels is shown in Figure 13. 


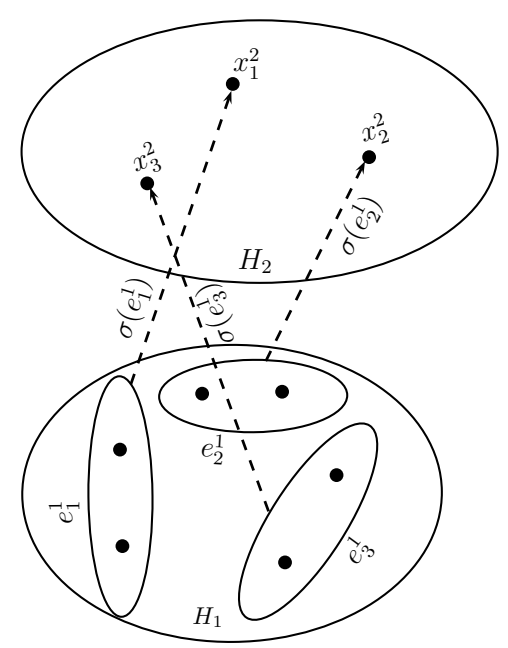

Figure 13. The zoom-out operator.

Theorem 4. Let $\sigma: H_{1} \rightarrow H_{2}$ be the zoom-out operator from $H_{1}=\left(X_{1}, E_{1}\right)$ to $H_{2}=\left(X_{2}, E_{2}\right)$, and let $E_{1}^{\prime} \subseteq E_{1}$. Then, the zoom-out operator $\sigma$ satisfies,

(i) $\sigma(\varnothing)=\varnothing$.

(ii) $\sigma$ maps the set of hyperedges of $H_{1}$ onto the set of vertices of $H_{2}$, i.e., $\sigma\left(E_{1}\right)=X_{2}$.

(iii) $\sigma\left(\left[E_{1}^{\prime}\right]^{c}\right)=\left[\sigma\left(E_{1}^{\prime}\right)\right]^{c}$.

Proof. (i) This part is trivially satisfied.

(ii) By applying the definition of $\sigma$, we have $\sigma\left(e_{i}^{1}\right)=x_{i}^{2}$. Since the hyperedges define a partition of hypergraph, we have $E_{1}=\left\{e_{1}^{1}, e_{2}^{1}, e_{3}^{1}, \cdots, e_{n}^{1}\right\}=\bigcup_{e_{i}^{1} \in E_{1}} e_{i}^{1}$. Then,

$\sigma\left(E_{1}\right)=\sigma\left(\bigcup_{e_{i}^{1} \in E_{1}} e_{i}^{1}\right)=\bigcup_{e_{i}^{1} \in E_{1}} \sigma\left(e_{i}^{1}\right)=\bigcup_{x_{i}^{2} \in X_{2}} x_{i}^{2}=X_{2}$.

(iii) Assume that $\left[E_{1}^{\prime}\right]^{c}=V_{1}^{\prime}$, then it is obvious that $E_{1}^{\prime} \cap V_{1}^{\prime}=\varnothing$ and $E_{1}^{\prime} \cup V_{1}^{\prime}=E_{1}$. Suppose on the contrary that there exists at least one vertex $x_{i}^{2} \in \sigma\left(\left[E_{1}^{\prime}\right]^{c}\right)$, but $x_{i}^{2} \notin\left[\sigma\left(E_{1}^{\prime}\right)\right]^{c} . x_{i}^{2} \in \sigma\left(\left[E_{1}^{\prime}\right]^{c}\right)$ implies that $x_{i}^{2} \in \sigma\left(V_{1}^{\prime}\right) \Rightarrow x_{i}^{2} \in \underset{e_{i}^{1} \in V_{1}^{\prime}}{\bigcup} \sigma\left(e_{i}^{1}\right) \Rightarrow x_{i}^{2} \in \underset{e_{i}^{1} \in E_{1} \backslash E_{1}^{\prime}}{\bigcup} \sigma\left(e_{i}^{1}\right)$. Since $x_{i}^{2} \notin\left[\sigma\left(E_{1}^{\prime}\right)\right]^{c} \Rightarrow x_{i}^{2} \in \sigma\left(E_{1}^{\prime}\right) \Rightarrow$ $x_{i}^{2} \in \bigcup_{e_{i}^{1} \in E_{1}^{\prime}} \sigma\left(e_{i}^{1}\right)$, which is contradiction to our assumption. Hence, $\sigma\left(\left[E_{1}^{\prime}\right]^{c}\right)=\left[\sigma\left(E_{1}^{\prime}\right)\right]^{c}$.

Definition 34. Let $H_{1}=\left(X_{1}, E_{1}\right)$ and $H_{2}=\left(X_{2}, E_{2}\right)$ be two levels of $q$-RPFHs, and $H_{1}$ possesses a stronger granularity than $H_{2}$. Let $E_{1}^{\prime} \subseteq E_{1}$, then $\hat{\sigma}\left(E_{1}^{\prime}\right)=\left\{e_{i}^{2} \mid e_{i}^{2} \in E_{2}, \kappa\left(e_{i}^{2}\right) \subseteq E_{1}^{\prime}\right\}$ is called the internal zoom-out operator.

The operator $\check{\sigma}\left(E_{1}^{\prime}\right)=\left\{e_{i}^{2} \mid e_{i}^{2} \in E_{2}, \kappa\left(e_{i}^{2}\right) \cap E_{1}^{\prime} \neq \varnothing\right\}$ is called the external zoom-out operator.

Example 8. Let $H_{1}=\left(X_{1}, E_{1}\right)$ and $H_{2}=\left(X_{2}, E_{2}\right)$ be two levels of q-RPFHs, and $H_{1}$ possesses a stronger granularity than $H_{2}$, where $E_{1}=\left\{e_{1}^{1}, e_{2}^{1}, e_{3}^{1}, e_{4}^{1}, e_{5}^{1}, e_{6}^{1}\right\}$ and $E_{2}=\left\{e_{1}^{2}, e_{2}^{2}, e_{3}^{2}\right\}$. Furthermore, $e_{1}^{2}=\left\{x_{1}^{2}, x_{3}^{2}\right\}, e_{2}^{2}=\left\{x_{2}^{2}, x_{4}^{2}\right\}, e_{3}^{2}=\left\{x_{5}^{2}, x_{6}^{2}\right\}$, as shown in Figure 14.

Let $E_{1}^{\prime}=\left\{e_{2}^{1}, e_{3}^{1}, e_{4}^{1}, e_{5}^{1}\right\}$ be the subset of hyperedges of $H_{1}$, then we cannot zoom-out to $H_{2}$ directly; thus, by using the internal and external zoom-out operators, we have the following relations.

$\hat{\sigma}\left(\left\{e_{2}^{1}, e_{3}^{1}, e_{4}^{1}, e_{5}^{1}\right\}\right)=\left\{e_{2}^{2}\right\}$,

$\breve{\sigma}\left(\left\{e_{2}^{1}, e_{3}^{1}, e_{4}^{1}, e_{5}^{1}\right\}\right)=\left\{e_{1}^{2}, e_{2}^{2}, e_{3}^{2}\right\}$. 


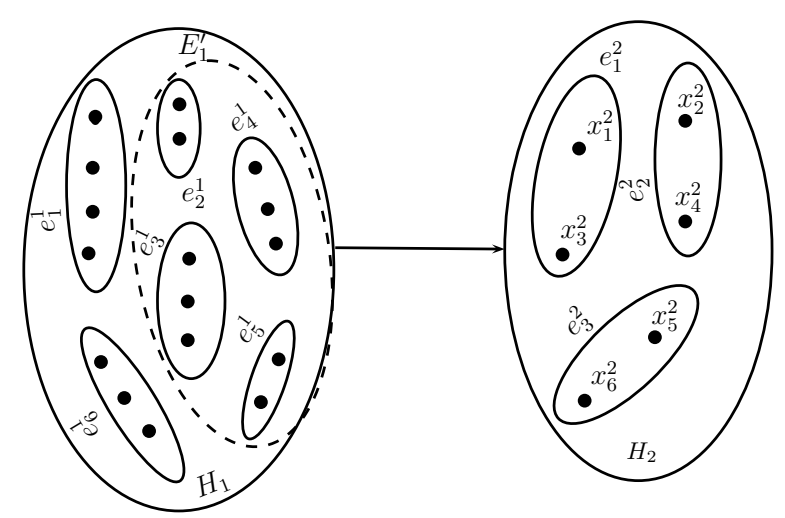

Figure 14. The internal and external zoom-out operators.

\section{Comparison Analysis}

A $q$-ROFS is defined as an ordered pair $(T, F)$ such that the sum of the $q$ th power of the positive degree $T$ and the $q$ th power of negative degree $F$ is no greater than one, i.e., $T^{q}+F^{q} \leq 1$. Note that IFSs and PYFSs are special cases of $q$-ROFSs, i.e, every IF grade is also a PYF grade, as well as a $q$-ROF grade. Suppose that $(x, y)$ is an IF grade, where $x \in[0,1], y \in[0,1]$, and $0 \leq x+y \leq 1$, since $x^{q} \leq x, y^{q} \leq y, q \geq 1$, so we have $0 \leq x^{q}+y^{q} \leq 1$. This implies that the class of $q$-ROFSs generalizes the classes of IFSs and PYFSs. In spite of the fact that IFSs, $q$-ROFSs, and PYFSs are applicable effectively in many decision making problems, still there are various circumstances that are difficult to handle using these models. For example, voters can be classified into four groups of people who: vote for, abstain, or refuse in a voting system, i.e., in many real-life situations, there are many answers other than yes or no, including, abstain and refusal. In such cases, the existing models of IFSs, $q$-ROFSs, and PYFSs fail to work effectively. Thus, Cuong [16] gave the idea of PFS, which is characterized by a positive degree $T$, a neutral degree $N$, and a negative degree $F$ such that $T+N+F \leq 1$. We have proposed a model of GrC based on $q$-RPFH and combined the advantages of two fruitful theories of $q$-ROFSs and PFSs. Our proposed $q$-RPF $(T, N, F)$ model not only expresses the neutral degree, but also relaxes the condition of PFS that the sum of the three degrees must be less than one since $T^{q}+N^{q}+F^{q} \leq 1, q \geq 1$ is the defining constraint of $q$-RPFS. Note that the proposed model extends the $q$-ROF and PF models by considering the neutral degree and by taking the constraint $T^{q}+N^{q}+F^{q} \leq 1, q \geq 1$ into consideration; for example, if a decision maker assigns the positive, neutral, and negative degrees as $0.6,0.3$, and 0.5 , respectively. Then, the triplet $(0.6,0.3,0.5)$ cannot be handled through $q$-ROF or PF models, whereas the proposed $q$-RPF model can deal with this problem. This reveals that the $q$-RPF model contains a higher capacity in modeling of uncertain data than the $q$-ROF and PF models. It is worth noting that as we increase the value of parameter $q$, the space of uncertain data also increases, and the bounding constraint is satisfied by more triplets. Thus, a wider range of vague information can be expressed using $q$-RPFSs. Our proposed model is more generic as compared to the $q$-ROF and PF models, as when $q=1$, the model reduces to the PF model, and when $N=0, q \geq 1$, it reduces to the $q$-ROF model. Hence, our approach is more flexible and generalized, and different values of $q$ can be chosen by decision makers according to the different attitudes.

\section{Conclusions and Future Directions}

A $q$-rung picture fuzzy set is the generalization of the picture of the fuzzy set and the $q$-rung orthopair fuzzy set. This model deals with uncertainty and vagueness more practically and effectively because of its third component, i.e., the neutral part. The proposed $q$-rung picture fuzzy model owns the advantages of the $q$-rung orthopair fuzzy set, as well as the picture fuzzy set and proves to be a more powerful framework to analyze the qualitative and quantitative studies. Granular computing is a general criterion that emphasizes the exploitation of multi-level granular structures. GrC reflects and captures our ability to explore real-world problems at different levels of granularity and enables us 
to change these granularities during problem solving. In this paper, we have combined the $q$-RPFSs with hypergraphs to attain the advantages of both theories. A $q$-RPFS is the generalization of PFS and $q$-ROFS. This set deals with uncertainty and vagueness more practically and effectively because of its third component, i.e., the neutral part. It is worth noting that as we increase the value of parameter $q$, the space of uncertain data also increases, and the bounding constraint is satisfied by more triplets. Thus, a wider range of vague information can be expressed by using $q$-RPFSs. Our proposed model is more generic as compared to $q$-ROF and PF models, as when $q=1$, the model reduces to the PF model, and when $N=0, q \geq 1$, it reduces to $q$-ROF model. We have defined $q$-RPFHs, and then, the proposed model has been used to examine the $\mathrm{GrC}$. In this hypergraph model, we have obtained the granules as hyperedges from $q$-RPFER, and the partition of the universe has been defined by means of these granules. Further, we have defined the zoom-in and zoom-out operators to give the description of mappings between two hypergraphs. We have also discussed a concrete example to reveal the validity and applicability of our proposed model. We aim to extend our research: (1) q-rung picture fuzzy directed hypergraphs, (2) fuzzy rough soft directed hypergraphs, (3) granular computing based on fuzzy rough hypergraphs, (4) hesitant $q$-rung orthopair fuzzy hypergraphs, and (5) $q$-rung picture fuzzy concept lattice.

Author Contributions: A.L., M.A., and A.N.A.K. conceived of the presented concept. A.L. and M.A. developed the theory and performed the computations. A.N.A.K. verified the analytical methods.

Funding: This research received no external funding

Acknowledgments: The authors are grateful to the editor of the journal and the anonymous referees for their valuable comments.

Conflicts of Interest: The authors declare no conflict of interest.

\section{References}

1. Lin, T.Y. Granular computing: From rough sets and neighborhood systems to information granulation and computing with words. In Proceedings of the European Congress on Intelligent Techniques and Soft Computing, Aachen, Germany, 8-12 September 1997.

2. Zhang, L.; Zhang, B. Hierarchy and Multi-Granular Computing, Quotient Space based Problem Solving; Tsinghua University Press: Beijing, China, 2014; pp. 45-103.

3. Yang, J.; Wang, G.; Zhang, Q. Knowledge distance measure in multigranulation spaces of fuzzy equivalence relation. Inf. Sci. 2018, 448, 18-35. [CrossRef]

4. Stell, J.G. Granulation for graphs. In Proceedings of the International Conference on Spatial Information Theory, Stade, Germany, 25-29 August 1999; pp. 417-432.

5. Chen, G.; Zhong, N. Granular structures in graphs. In Proceedings of the International Conference on Rough Sets and Knowledge Technology, Banff, AB, Canada, 9-12 October 2011; pp. 649-658.

6. Chiaselotti, G.; Ciucci, D.; Gentile, T. Simple graphs in granular computing. Inf. Sci. 2016, 340, $279-304$. [CrossRef]

7. Bisi, C.; Chiaselotti, G.; Ciucci, D.; Gentile, T.; Infusino, F.G. Micro and macro models of granular computing induced by the indiscernibility relation. Inf. Sci. 2017, 388, 247-273. [CrossRef]

8. Berge, C. Graphs and Hypergraphs; North-Holland Publishing Company: Amsterdam, The Netherlands, 1973.

9. Liu, Q.; Jin, W.B.; Wu, S.Y.; Zhou, Y.H. Clustering research using dynamic modeling based on granular computing. In Proceedings of the IEEE International Conference on Granular Computing, Beijing, China, 25-27 July 2005; pp. 539-543.

10. Wong, S.K.M.; Wu, D. Automated mining of granular database scheme. In Proceedings of the IEEE International Conference on Fuzzy Systems, St. Louis, MO, USA, 25-28 May 2002; pp. 690-694.

11. Chen, G.; Zhong, N.; Yao, Y. A hypergraph model of granular computing. In Proceedings of the IEEE International Conference on Granular Computing, Hangzhou, China, 26-28 August 2008; pp. 130-135.

12. Zadeh, L.A. Fuzzy sets. Inf. Control. 1965, 8, 338-353. [CrossRef]

13. Atanassov, K. Intuitionistic fuzzy sets. Fuzzy Sets Syst. 1989, 33, 37-45. [CrossRef] 
14. Yager,R.R. Pythagorean fuzzy subsets. In Proceedings of the Joint IFSA World Congress and NAIFPS Annual Meeting, Edmonton, AB, Canada, 24-28 June 2013; pp. 57-61.

15. Yager, R.R. Generalized orthopair fuzzy sets. IEEE Trans. Fuzzy Syst. 2017, 25, 1222-1230. [CrossRef]

16. Cuong, B.C. Picture fuzzy sets. J. Comput. Sci. Cybern. 2014, 30, 409-420.

17. Li, L.; Zhang, R.; Wang, J.; Shang, X.; Bai, K. A novel approach to multi-attribute group decision-making with $q$-rung picture linguistic information. Symmetry 2018, 10, 172. [CrossRef]

18. Akram, M.; Habib, A.; Ilyas, F.; Dar, J.M. Specific types of Pythagorean fuzzy graphs and application to decision-making. Math. Computat. Appl. 2018, 23, 42. [CrossRef]

19. Akram, M.; Habib, A.; Koam, A.N.A. A novel description on edge-regular $q$-rung picture fuzzy graphs with application. Symmetry 2019, 11, 489. [CrossRef]

20. Kaufmann, A. Introduction a la Thiorie des Sous-Ensemble Flous; Masson: Paris, France, 1977.

21. Lee-kwang, H.; Lee, K.-M. Fuzzy hypergraph and fuzzy partition. IEEE Trans. Syst. Man Cybern. 1995, 25, 196-201. [CrossRef]

22. Mordeson, J.N.; Nair, P.S. Fuzzy Graphs and Fuzzy Hypergraphs, 2nd ed.; Physica Verlag: Heidelberg, Germany, 2001.

23. Wang, Q.; Gong, Z. An application of fuzzy hypergraphs and hypergraphs in granular computing. Inf. Sci. 2018, 429, 296-314. [CrossRef]

24. Gong, Z.; Wang, Q. On the connection of fuzzy hypergraph with fuzzy information system and fuzzy concept lattice. J. Intell. Fuzzy Syst. 2017, 33, 1665-1676. [CrossRef]

25. Parvathi, R.; Thilagavathi, S.; Karunambigai, M.G. Intuitionistic fuzzy hypergraphs. Cybern. Inf. Technol. 2009, 9, 46-53.

26. Akram, M.; Dudek, W.A. Intuitionistic fuzzy hypergraphs with applications. Inf. Sci. 2013, 218, 182-193. [CrossRef]

27. Akram, M.; Luqman, A. Intuitionistic single-valued neutrosophic hypergraphs. OPSEARCH 2017, 54, 799-815. [CrossRef]

28. Akram, M.; Luqman, A. Certain concepts of bipolar fuzzy directed hypergraphs. Mathematics 2017, 5, 17. [CrossRef]

29. Luqman, A.; Akram, M.; Kenani, A.L.A. q-Rung orthopair fuzzy hypergraphs with applications. Mathematics 2019, 7, 260. [CrossRef]

30. Luqman, A.; Akram, M.;Koam, A.N. An m-polar fuzzy hypergraph model of granular computing. Symmetry 2019, 11, 483. [CrossRef]

31. Zadeh, L.A. Similarity relations and fuzzy orderings. Inf. Sci. 1971, 3, 177-200. [CrossRef]

32. Zhang,L.; Zhang, B. The Theory and Applications of Problem Solving-Quotient Space based Granular Computing, 2nd ed.; Tsinghua University Press: Beijing, China, 2007.

33. Zhang,L.; Zhang, B. The structural analysis of fuzzy sets. J. Approx. Reason. 2005, 40, 92-108. [CrossRef]

34. Yao, Y.Y. A partition model of granular computing. In Transactions on Rough Sets I. Lecture Notes in Computer Science; Peters, J.F., Skowron, A., Grzymała-Busse, J.W., Kostek, B., Świniarski, R.W., Szczuka, M.S., Eds.; Springer: Berlin, Germany, 2004; Volume 3100, pp. 232-253.

(C) 2019 by the authors. Licensee MDPI, Basel, Switzerland. This article is an open access article distributed under the terms and conditions of the Creative Commons Attribution (CC BY) license (http://creativecommons.org/licenses/by/4.0/). 